\title{
Room Temperature Metallic Conductivity in a Metal-Organic Framework Induced by Oxidation
}

DOI:

10.1021/jacs.9b06898

\section{Document Version}

Accepted author manuscript

Link to publication record in Manchester Research Explorer

\section{Citation for published version (APA):}

Clough, A. J., Orchanian, N. M., Skelton, J. M., Neer, A. J., Howard, S. A., Downes, C. A., Piper, L. F. J., Walsh, A., Melot, B. C., \& Marinescu, S. C. (2019). Room Temperature Metallic Conductivity in a Metal-Organic Framework Induced by Oxidation. Journal of the American Chemical Society, 141(41), 16323-16330. https://doi.org/10.1021/jacs.9b06898

\section{Published in:}

Journal of the American Chemical Society

\section{Citing this paper}

Please note that where the full-text provided on Manchester Research Explorer is the Author Accepted Manuscript or Proof version this may differ from the final Published version. If citing, it is advised that you check and use the publisher's definitive version.

\section{General rights}

Copyright and moral rights for the publications made accessible in the Research Explorer are retained by the authors and/or other copyright owners and it is a condition of accessing publications that users recognise and abide by the legal requirements associated with these rights.

\section{Takedown policy}

If you believe that this document breaches copyright please refer to the University of Manchester's Takedown Procedures [http://man.ac.uk/04Y6Bo] or contact uml.scholarlycommunications@manchester.ac.uk providing relevant details, so we can investigate your claim.

\section{OPEN ACCESS}




\title{
Room Temperature Metallic Conductivity in a Metal-Organic Framework Induced by Oxidation
}

\author{
Andrew J. Clough, ${ }^{a}$ Nicholas M. Orchanian, ${ }^{a}$ Jonathan M. Skelton, ${ }^{\mathrm{b}}$ Abbey J. Neer, ${ }^{\mathrm{a}}$ Sebastian A. \\ Howard, ${ }^{\mathrm{c}}$ Courtney A. Downes, ${ }^{\mathrm{a}}$ Louis F. J. Piper, ${ }^{\mathrm{c}, \mathrm{d}}$ Aron Walsh, ${ }^{\mathrm{e}, \mathrm{f}}$ Brent C. Melot, ${ }^{\text {a* }}$ and Smaranda C. \\ Marinescu ${ }^{\mathrm{a} *}$ \\ aDepartment of Chemistry, University of Southern California, Los Angeles, California 90089, United States

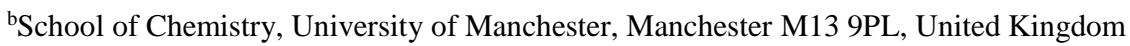 \\ ${ }^{\mathrm{c}}$ Department of Physics, Applied Physics and Astronomy, Binghamton University, Binghamton, New York 13902, United States \\ ${ }^{\mathrm{d}}$ Materials Science \& Engineering, Binghamton University, Binghamton, New York 13902, United States \\ 'Department of Materials, Imperial College London, London SW7 2AZ, United Kingdom \\ fDepartment of Materials Science and Engineering, Yonsei University, Seoul 03722, Korea
}

\begin{abstract}
Metal-organic frameworks (MOFs) containing redox active linkers have led to hybrid compounds exhibiting high electrical conductivity, which enables their use in applications in electronics and electrocatalysis. While many computational studies predict two-dimensional (2D) MOFs to be metallic, the majority of experiments show decreasing conductivity on cooling, indicative of a gap in the electronic band structure. To date, only a handful of MOFs have been reported that exhibit increased electrical conductivity upon cooling indicative of a metallic character, which highlights the need for better understanding the origin of the conductivity. A 2D MOF containing iron bis(dithiolene) motifs was recently reported to exhibit semiconducting behavior with record carrier mobility. Herein, we report that high crystallinity and the elimination of guest species results in an iron 2,3,6,7,10,11-tripheylenehexathiolate (THT) MOF, FeTHT, exhibiting a complex transition from semiconducting to metallic upon cooling, similar to what was shown for the analogous CoTHT. Remarkably, exposing the FeTHT to air significantly influences the semiconducting-to-metallic transition temperature (100 to $300 \mathrm{~K}$ ), and ultimately results in a material showing metallic-like character at, and above, room temperature. This study indicates these materials can tolerate a substantial degree of doping that ultimately results in charge delocalization and metallic-like conductivity, an important step towards enabling their use in chemiresistive sensing and optoelectronics.
\end{abstract}

\section{INTRODUCTION}

Metal-organic frameworks (MOFs) are crystalline nanoporous materials composed of metal ions or clusters linked by organic ligands. ${ }^{1-4}$ The hybrid organic/inorganic nature of MOFs allows for synthetic tunability, leading to MOFs with varying pore sizes and chemical environments, and therefore, different physical and chemical properties. ${ }^{1-5}$ These properties have led to applications primarily in gas storage and separation ${ }^{6-8}$ and catalysis, ${ }^{9}$ which take advantage of the inherent porosity and high surface area of these materials. ${ }^{10}$ The use of MOFs in technologies that require charge transport, such as electronics ${ }^{11,12}$ and electrocatalysis, ${ }^{13}$ has lagged due to their generally poor electrical conductivity. The weak covalent overlap between the metal and ligand orbitals results in localized (small band width) electronic band structures with low mobility charge 
carriers, preventing fast charge transport through the framework. This leads to materials with insulating or large gap semiconducting behavior.,14,15 Efforts to reduce the barriers to charge transport have included the addition of guest species, ${ }^{16-18}$ doping, ${ }^{19-21}$ and variation of the metal center and its oxidation state..$^{22-26}$ These modifications can encourage through-space ${ }^{27,28}$ or throughbond ${ }^{29}$ electronic transport and have led to MOFs with improved conductivities, with one example reporting tunable conductivity over six orders of magnitude. ${ }^{17}$

Recently, the development of MOFs with redox active linkers has led to a breakthrough in the field of electrically conductive MOFs. ${ }^{11,12,15,30-34}$ Several two- and three-dimensional (2D/3D) frameworks with planar, $\pi$-conjugated, and redox-active linkers, like semiquinones/cathecolates, ${ }^{35-43}$ diimines, ${ }^{44-48}$ and dithiolenes, ${ }^{19,21,48-61}$ have been reported to display high electrical conductivity. Yet, while computational studies often predict these 2D MOFs to be metallic, ${ }^{50,62-65}$ the majority of the frameworks reported display a decrease in conductivity on cooling as thermally-populated carriers are lost. In contrast, the primary mechanism for carrier scattering in metals is due to lattice vibrations that are significantly dampened at lower temperatures, resulting in more efficient transport on cooling. We previously reported the temperature-dependent electrical conductivity of a $2 \mathrm{D}$ cobalt 2,3,6,7,10,11tripheylenehexathiolate (THT) MOF. ${ }^{52}$ While the measured conductivity at $300 \mathrm{~K}$ was modest $\left(3.2 \times 10^{-2} \mathrm{~S} / \mathrm{cm}\right)$, a complex transition from semiconducting to metallic behavior was observed on cooling. This represented the first direct experimental observation of a MOF exhibiting metallic conductivity. The metallic character was corroborated by density-functional theory (DFT) calculations, which predicted semimetallic behavior through electronic states arising from interlayer overlap of metal $d$ and ligand $p$ orbitals. This complex behavior was attributed to a convolution of thermal expansion along the $c$-direction, stacking faults leading to misalignment of the layers, and significantly different transport at the grain-boundaries.

Recently, an analogous iron THT MOF with ammonium charge-compensating cations, $\left(\mathrm{Fe}_{3}(\mathrm{THT})_{2}\left(\mathrm{NH}_{4}\right)_{3}\right)$, was studied using high-frequency terahertz photoconductivity and Hall effect measurements. The reported $\mathrm{Fe}_{3}(\mathrm{THT})_{2}\left(\mathrm{NH}_{4}\right)_{3}$ material displays semiconducting behavior with record room-temperature carrier mobilities. ${ }^{55}$ The calculated band structure, which explicitly included ammonium counterions, predicts semiconducting behavior with a bandgap of $\sim 350 \mathrm{meV}$. Strong orbital hybridization is observed between the $d$ orbitals of Fe, the bis(dithiolene) moieties, and the triphenylene units. While promising room-temperature mobilities were reported for this material, the reported temperature-dependent resistivity studies indicate a decrease in conductivity on cooling, in contrast to the cobalt analogue. ${ }^{51}$ This discrepancy emphasizes the current knowledge gap in understanding the nature of charge transport in MOFs. This shortcoming is especially noteworthy in materials reported to date using benzenehexathiolate and hexaiminobenzene linkers, which display decreases in their conductivity on cooling, despite ultraviolet photoelectron spectroscopy (UPS) studies revealing Fermi edges that strongly suggest metallic character. ${ }^{46,50,58}$

Related studies of a copper benzenehexathiolate (CuBHT) MOF report a decrease in conductivity on cooling, consistent with semiconducting behavior. ${ }^{49}$ Using a modified synthetic procedure, the crystallinity of the resulting CuBHT was improved as evidenced by powder X-ray 
diffraction (PXRD) studies. ${ }^{61}$ Temperature-dependent resistivity studies of the crystalline MOF indicate a decrease in resistivity upon cooling, as expected for a metal, followed by the noteworthy observation of a superconducting transition at low temperatures. ${ }^{61}$ These studies highlight the influence of crystallinity in dictating the transport properties of 2D MOFs (semiconducting vs. metallic). Yet, ideally, the transition from semiconducting to metallic behavior would be controllable through chemical and post-synthetic modification rather than a reliance on single-crystal growth, which remains a substantial challenge in the field. As a promising example of this goal, the chemical reduction of an analogous silver benzenehexathiolate (AgBHT) 2D MOF was shown by UPS studies to eliminate the Fermi edge of the pristine material, suggesting a metallic-to-semiconducting transition, with a corresponding reduction $(\sim 3,500$-fold $)$ in the electrical conductivity. ${ }^{58}$

Motivated by the knowledge that metal identity, doping, and the presence of guest species all have significant influence on the electronic properties of metal bis(dithiolene) species and related 2D MOFs, ${ }^{66-68}$ we present a modified synthetic procedure to prepare the FeTHT framework as a comparative analogue to CoTHT. This modified protocol is informed by DFT predictions and seeks to circumvent the inclusion of ammonium guest species and generate materials with improved crystallinity as a means of inducing the semiconducting-to-metallic transition observed in CoTHT. Temperature-dependent resistivity studies of the as-prepared FeTHT framework confirm a transition from semiconducting to metallic upon cooling, which is analogous to the behavior observed for the CoTHT system. ${ }^{52}$ We further explore the effect that exposure to oxygen has on the conductivity of the framework and show that oxidation of the sample leads to an increase in the semiconducting-to-metallic transition temperature as a function of exposure, resulting in a material with metallic-like character at room temperature. Using a combination of X-ray photoelectron spectroscopy (XPS) and magnetic studies, we confirm that both the metal center and the ligand scaffold in FeTHT are oxidized. These results show that the semiconducting-to-metallic transition temperature is highly sensitive to dopant concentrations and suggests that the development of controlled post-synthetic redox treatments could be effective at enhancing the functional properties of this family of materials.

\section{RESULTS AND DISCUSSION Synthesis and Characterization}

The 2D FeTHT MOF was synthesized using a liquid-liquid interfacial reaction as previously reported for the cobalt analogue (Figure 1). ${ }^{52,69}$ The trinucleating ligand scaffold, triphenylene-2,3,6,7,10,11-hexathiol, was treated with N-Methyl-2-pyrrolidone (NMP) in an ethyl acetate solution and sonicated. The generated suspension was gently layered onto an aqueous solution of the Fe(II) chloride, leading to the formation of a black film of FeTHT at the liquidliquid interface over the course of 5 days. The black film was then deposited on to glass substrates as thin films or collected as a powder for bulk measurements. This synthetic methodology is in contrast to that reported recently for $\mathrm{Fe}_{3}(\mathrm{THT})_{2}\left(\mathrm{NH}_{4}\right)_{3}$, where a solution of $\mathrm{Fe}(\mathrm{acac})_{2}$ in chloroform was layered with an aqueous solution of THT ligand with $\mathrm{NH}_{4} \mathrm{OH}$ as base. ${ }^{55}$ Since our synthetic procedure does not include $\mathrm{NH}_{4} \mathrm{OH}$, the variations employed here serve to exclude the $\mathrm{NH}_{4}{ }^{+}$guest species. This approach provides a platform for demonstrating the influence of guest 
species on bulk transport properties of the Fe system, as well as appropriately comparing the electronic behavior of the Fe and Co systems.

The crystallinity of the FeTHT framework was confirmed by PXRD using synchrotron radiation. FeTHT displays prominent peaks at $1.21^{\circ}, 2.18^{\circ}, 2.42^{\circ}, 3.21^{\circ}$, and a broad peak at $7.09^{\circ}$ (Figure 1). These peaks are similar to the ones reported for the CoTHT framework, suggesting analogous structural environments for the Co and Fe systems. ${ }^{52,69}$ The peak at $1.21^{\circ}$ corresponds to the [100] reflection and is indicative of a pore diameter of approximately $2.0 \mathrm{~nm}$. This pore diameter is consistent with the recently reported PXRD pattern of $\mathrm{Fe}_{3}(\mathrm{THT})_{2}\left(\mathrm{NH}_{4}\right)_{3}$ $(\sim 1.9 \mathrm{~nm})$, confirming that the modified synthetic procedure employed here enables exclusion of the ammonium guest species without substantially altering the $2 \mathrm{D}$ structure of the material. ${ }^{55}$ Figure 1 illustrates a comparison of the experimentally observed diffraction pattern of FeTHT with the simulated pattern of a model using the $P 6 / \mathrm{mmm}$ space group and unit cell parameters of $a$ $=b=22.52 \AA$ and $c=3.34 \AA$ (Figure S1). This diffraction data indicates good long-range order in the $a b$ plane and weak ordering along the $c$ axis. The morphology of FeTHT films was examined using scanning electron microscopy (SEM). Low magnification images (Figure 1, inset) show few cracks, and higher magnification images (Figure S2) reveal flat, sheet-like morphologies consistent with images of similar 2D MOFs. Nitrogen gas sorption isotherms show that FeTHT has a Brunauer-Emmett Teller (BET) surface area of $441 \mathrm{~m}^{2} / \mathrm{g}$ (Figure S3), which is similar to the surface areas reported for analogous cobalt and iron THT frameworks of 370 and $526 \mathrm{~m}^{2} / \mathrm{g}$, respectively. ${ }^{52,55}$
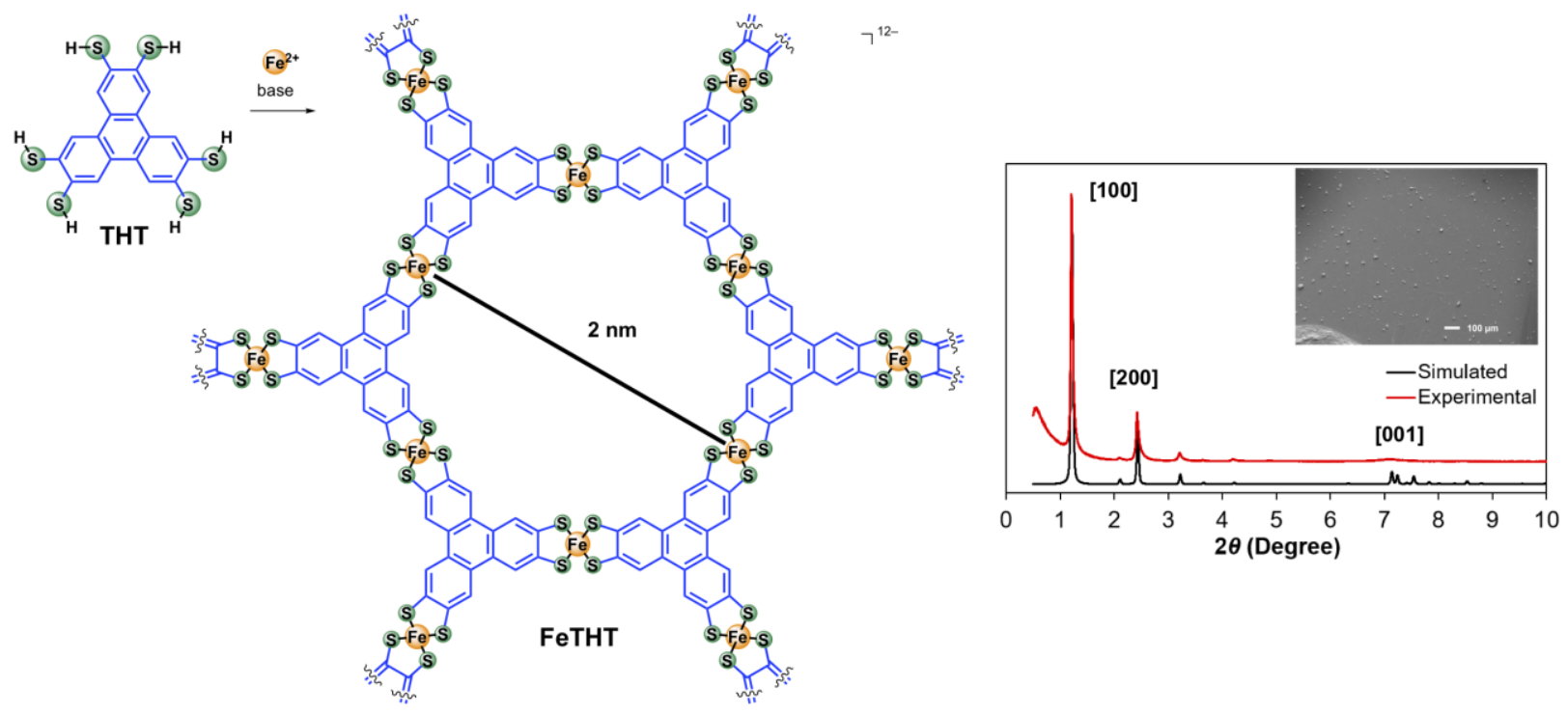

Figure 1. Synthesis and structure of the FeTHT framework. The plot shows a comparison of the experimental (red; synchrotron radiation with $\lambda=0.414576 \AA$ ) and simulated (black) PXRD patterns, and the inset shows an SEM image at $75 \times$ low magnification.

Following exposure of the frameworks to air $\left(3\right.$ days at $\left.60{ }^{\circ} \mathrm{C}\right)$, the PXRD pattern of FeTHT samples show the strong peaks at $1.21^{\circ}, 2.18^{\circ}, 2.42^{\circ}, 3.21^{\circ}$ in the as-prepared sample (Figures S4-S6) are maintained after exposure to the atmosphere. The broad peak at $7.09^{\circ}$ shifts to lower angles and sharpens to reveal features consistent with the simulated spectrum, suggesting 
more structural order in the $c$-direction. Upon cooling, a decrease in interlayer distance is observed for the [001] reflection (from $3.34 \AA$ to $3.23 \AA$ ), however the [100] reflection remains unchanged (Figure S5). No significant change in film thickness or morphology was observed by AFM after oxidation of the framework (Figure S7). The measured BET surface area of the oxidized FeTHT sample was determined to be $27.6 \mathrm{~m}^{2} / \mathrm{g}$ (Figure S8), indicating a significant loss of accessible surface area. A similar reduction in the available surface area from 370 to $50 \mathrm{~m}^{2} / \mathrm{g}$ was previously reported for $\mathrm{Fe}(\text { tri })_{2} \mathrm{MOF}$ upon oxidation, where tri $=1,2,3$-triazolate, and was attributed to occupation of the pores by charge-balancing species $\left(\mathrm{BF}_{4}^{-}\right){ }^{26}$

\section{Density Functional Theory (DFT) Calculations}

To understand the electronic structure of the FeTHT framework in the absence of ammonium guest species, first-principles calculations were carried out on the modelled structure using plane-wave DFT (see supporting information for details). The calculations predict a ground-state magnetization density with one unpaired electron per Fe ion, consistent with $\mathrm{Fe}^{3+}$ in a distorted-octahedral crystal field. A strong preference for ferromagnetic coupling between layers and a weaker frustrated antiferromagnetic coupling within the $2 \mathrm{D}$ sheets produces a net magnetic moment of $2.2 \mu_{\mathrm{B}}$ per unit cell. The predicted equilibrium geometry is in good agreement with the experimentally-measured lattice parameters, and similar results were obtained from $\mathrm{DFT}+U$ calculations with a Hubbard $U$ correction of $5 \mathrm{eV}$ applied to the Fe d orbitals.

As in our previous calculations on the analogous CoTHT framework, ${ }^{52}$ the band dispersion suggests that bulk FeTHT is a semi-metal with a small density of states at the Fermi energy (Figure 2). These states are primarily composed of Fe $d$ and $\mathrm{S} p$, with a strong interlayer interaction along the $c$-axis, corresponding to the $\Gamma-A$ segment of the band structure, which shows a significant dispersion of $\sim 1.8 \mathrm{eV}$. Carrier effective masses ranging from 0.55-6.72 $m_{e}$ were estimated from the band dispersion, which are comparable to the values of $0.29-8.04 m_{e}$ predicted for the CoTHT analogue. ${ }^{52}$ The band dispersion also shows a small number of metallic states along the $M$ - $\Gamma$ segment, corresponding to in-plane conductivity, with a carrier mass around $0.99 m_{e}$. Semi-metallic electronic structures were also predicted for both the FeTHT and CoTHT frameworks with DFT $+U$ calculations, which provide confidence that these results are not an artefact of unphysical delocalisation of the Fe $\mathrm{d}$ electrons. Prior electronic-structure calculations on the FeTHT framework with explicit ammonium guest molecules predict a narrow bandgap $(\sim 350 \mathrm{meV}),{ }^{55}$ highlighting the sensitivity of the electronic structure to subtle environmental factors. The predicted elimination of this gap upon removal of the $\mathrm{NH}_{4}{ }^{+}$provides a strong indication that the modified FeTHT reported here should exhibit distinct electronic behaviour relative to the previouslyreported semiconducting FeTHT. 


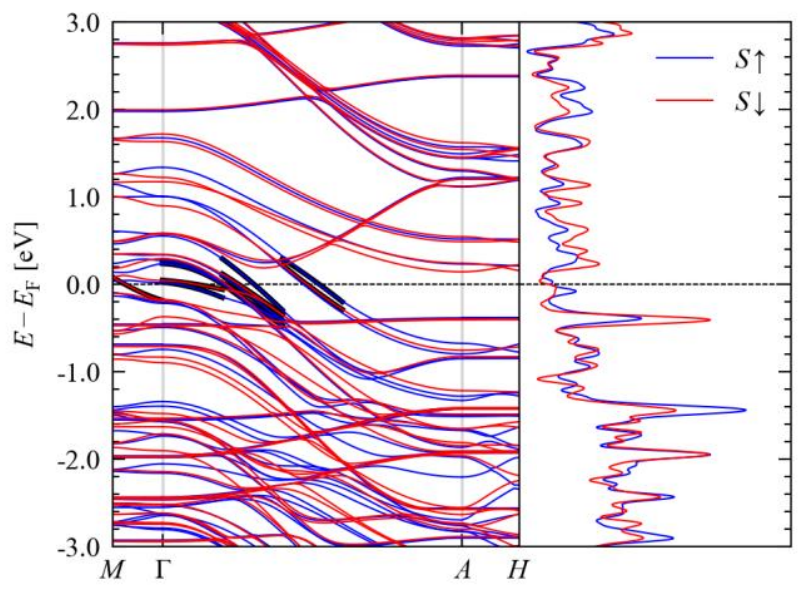

Figure 2. Calculated band dispersion and electronic density of states curves for the FeTHT framework with no guest species. The blue and red lines denote electronic states in the "up" and "down" spin channels. The thick black lines mark the regions of the dispersion used to evaluate $\partial^{2} E(k) / \partial k^{2}$ to estimate the carrier effective masses discussed in the text.

Computational studies of the $\mathrm{Ni}_{3}(\mathrm{HITP})_{2}$ framework ${ }^{44}$ reported that a staggered arrangement of the layers has the lowest total energy compared to an eclipsed geometry, whereas our previous calculations on the CoTHT framework found the latter to be lowest in energy. ${ }^{52}$ The calculated potentialenergy surface for stacking faults in FeTHT (Figure 3) shows that layer offsets of $\sim 1.0-1.25 \AA$ along the $a / b$ axis or a combined offset of $1.25 \AA$ along both axes are lower in energy than the eclipsed configuration by $\Delta E=83$ and $98 \mathrm{meV}$ per Fe ion, respectively. These small energy differences between the two configurations suggest that formation of stacking faults in FeTHT is likely to be somewhat facile, which is consistent with the broad [001] PXRD peak observed. Earlier calculations on the FeTHT framework with ammonium guest species suggest that an inclined AA-stacked geometry is the most stable, which is consistent with our results. ${ }^{55}$ As for the CoTHT framework, the calculations predict that the stacking faults would reduce the density of states around the Fermi energy and introduce a gap into the conduction band, both of which are intuitive given the nature of the metallic states. Intrinsic stacking faults, as well as environmental factors, may play key roles in defining the electronic structure of FeTHT and inducing a semiconductor-to-metal transition. 


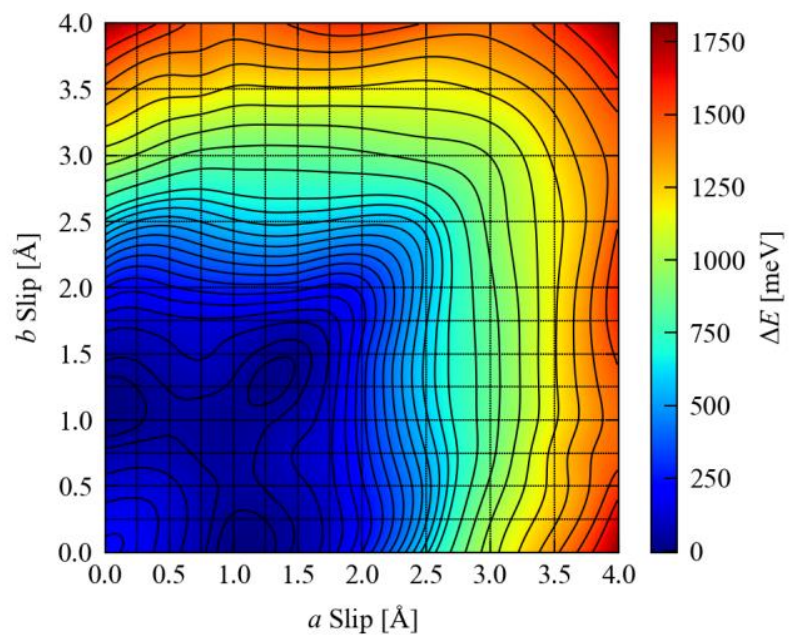

Figure 3. Calculated DFT potential-energy surface associated with layer offsets (stacking faults) in the pristine FeTHT framework.

\section{X-ray Photoelectron Spectroscopy (XPS) and Magnetism Studies}

The surface composition of the FeTHT films was investigated using X-ray photoelectron spectroscopy (XPS). Survey scans of pristine FeTHT films reveal the presence of Fe, S, C, and O. Fitting of the Fe $2 p$, shown in Figure $4 \mathrm{a}$, was primarily based on the Fe $2 p$ multiplet splitting of $\mathrm{Fe}_{3} \mathrm{O}_{4}$ which was used as a reference. ${ }^{70}$ The peaks at binding energies of 708.2, 709.2, and 710.3 $\mathrm{eV}$ are assigned to $\mathrm{Fe}^{2+}$, and the peaks at 710.1, 711.2, 712.3, and $713.5 \mathrm{eV}$ are assigned to $\mathrm{Fe}^{3+}$ (See Table S5). The Fe $2 \mathrm{p}$ region shows mixed $\mathrm{Fe}^{3+/ 2+}$ valency with approximately $52 \% \mathrm{Fe}^{2+}$ and $48 \% \mathrm{Fe}^{3+}$; indicating the presence of mixed oxidation states in pristine FeTHT. ${ }^{23}$ Figure $4 \mathrm{~b}$ shows peak fitting of the $S 2 p$ regions for the pristine sample. The binding energy difference of the $S 2 p$ $3 / 2-\mathrm{S} 2 \mathrm{p}_{1 / 2}$ doublet was fixed to $1.2 \mathrm{eV}$ with intensity ratio $2: 1 .^{71,72}$

Examination of the same XPS regions in a sample after prolonged air exposure reveals distinct changes in the $\mathrm{Fe} 2 p$ and $\mathrm{S} 2 p$ regions. Following seven days of exposure to ambient atmosphere, the first peak of the $\mathrm{Fe}^{2+}$ multiplet disappears and the experimental data can be fit by the $\mathrm{Fe}^{3+}$ multiplet alone (Figure 4a), indicating complete surface oxidation of the metal sites. In the S $2 p$ region, two new sets of peaks appear at higher binding energies of $\sim 166 \mathrm{eV}$ and $\sim 168 \mathrm{eV}$, indicating concomitant ligand oxidation (Figure 4b). These environments are attributed to sulfenate and sulfinate moieties, as previously reported in a palladium dithiolene molecular complex. ${ }^{73}$ To further substantiate these assignments, high resolution XPS of the corresponding $\mathrm{Pd}$ and Fe molecular species have been collected before and after $\mathrm{O}_{2}$ exposure (Figure $\mathrm{S} 18-\mathrm{S} 21$ ). The S $2 p$ region of the previously reported palladium coordination polymers confirms that aerobic oxidation results in a new oxidized sulphur-containing moiety, which appears at $\sim 167 \mathrm{eV}$ (Figures S20 and S21). ${ }^{73}$ This new feature was assigned as the added presence of sulfenate/sulfinate moieties, which was confirmed by the reported crystal structure of the resulting Pd polymer. ${ }^{73}$ Likewise, aerobic oxidation of the analogous iron dithiolene complex reproduces the additional $\mathrm{S}$ $2 p$ features observed for the FeTHT framework, which supports the assignment of these new 
features as sulfenate/sulfinate moieties (Figures S18 and S19). By examining the area of the peaks in the $\mathrm{O}_{2}$-exposed FeTHT sample (Figure 4b), approximately $49 \%$ of the thiolate moieties appear to be oxidized, with a sulfenate:sulfinate ratio of 1:4. These numbers are similar to the reported Pd dithiolene complex, in which half of all thiolates are oxidized, with a sulfenate/sulfinate ratio of $1: 2.3 .^{73}$

These XPS results thus confirm that both the metal center and the ligand backbone are oxidized after prolonged exposure to air. This is consistent with the predominant contributions from interacting $\mathrm{Fe}$ and $\mathrm{S}$ orbitals to the partially-occupied bands predicted by DFT. The calculations also reveal a preference for an offset rather than eclipsed layer stacking, indicating that interlayer Fe-S interactions may play a role in directing stacking manifolds. As these are expected to be weak interactions, based on the predicted ease of generating stacking faults, the formation of new S-O bonds upon oxidation may facilitate stronger Fe-O interlayer interactions, consistent with the sharpening of the peak at $7.09^{\circ}$ by synchrotron PXRD. As no such oxidized thiolate moieties were evident in the CoTHT material, we attribute the thickness-dependence of the cobalt system to a slower oxidation process relative to the analogous iron-containing framework. ${ }^{51}$
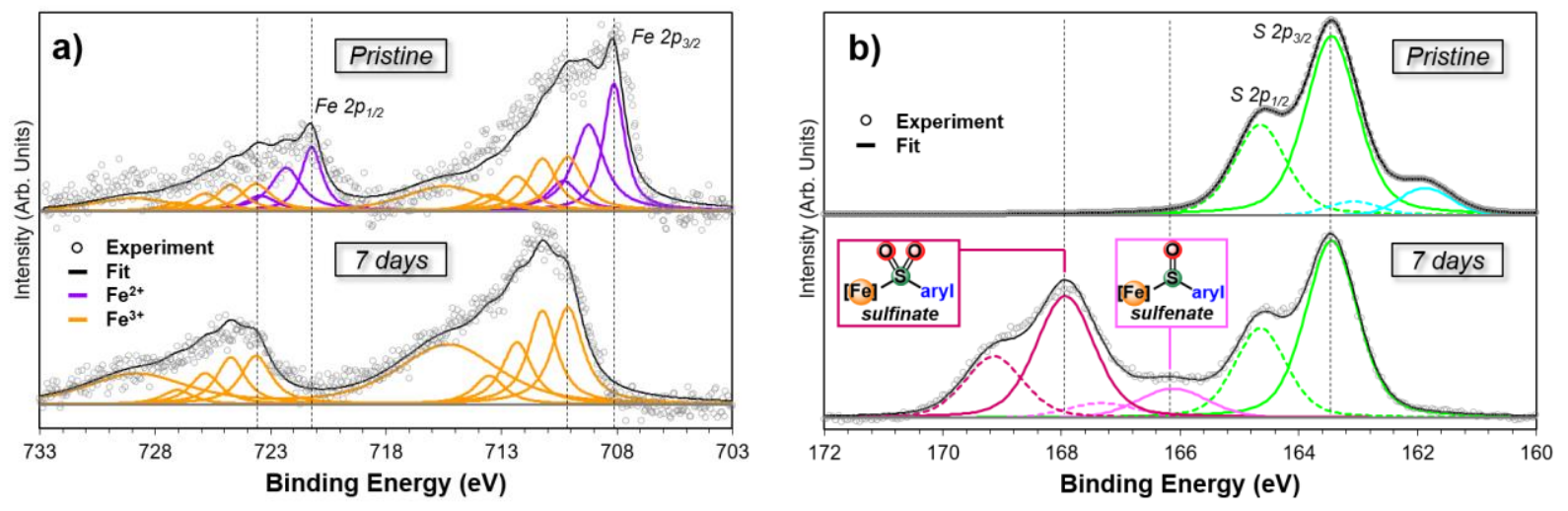

Figure 4. Fitting of the XPS spectra of pristine FeTHT and samples exposed to ambient atmosphere for 7 days. (a) Fe 2p region showing mixed valency $\left(\mathrm{Fe}^{2+/ 3+}\right)$ in pristine FeTHT; after exposure to air, only the $\mathrm{Fe}^{3+}$ peak is observed. (b) S $2 \mathrm{p}$ region in the pristine FeTHT; after exposure to air, two new doublets at $\sim 166 \mathrm{eV}$ (pink) and $\sim 168 \mathrm{eV}$ (burgundy) appear, in addition to the original features for the pristine FeTHT (green), indicating the formation of dithiolene moieties with different degrees of oxidation.

To evaluate the mixture of iron oxidation states in bulk FeTHT, magnetic measurements were performed. It was previously reported that mixed-valency in MOFs can enhance conductivity by promoting charge hopping. ${ }^{74}$ Recent studies of iron-based 3D MOFs have shown that controlling mixed-valency at $\mathrm{Fe}$ sites can have dramatic effects on the bulk transport properties, increasing conductivity by as many as eight orders of magnitude. ${ }^{25,26,75}$ By examining the saturation of unpaired spins in the hysteresis loop in FeTHT (Figure S22a), it is estimated that $33 \%$ of the iron centers are $\mathrm{Fe}^{3+}$, with the remaining $67 \% \mathrm{Fe}^{2+}$. These results are in agreement with the XPS studies, as similar mixed $\mathrm{Fe}^{3+/ 2+}$ valency is observed in the as-prepared FeTHT material, albeit a higher degree of oxidation is estimated by XPS (1:1 from XPS studies vs 2:1 
from magnetic studies). The higher degree of oxidation observed by XPS suggests that the oxidized species are concentrated towards the surface of the sample, as magnetic and XPS measurements analyse the bulk vs. surface composition, respectively. After exposure of the FeTHT to air for 3 days at $60^{\circ} \mathrm{C}$, the observed magnetic moment of the sample increases, which is consistent with oxidation to give an increased amount of $\mathrm{Fe}^{3+}$ (Figure S22b). This increased moment reflects an increase of the $\mathrm{Fe}^{2+} / \mathrm{Fe}^{3+}$ ratio from $2: 1$ to $1: 1$. The magnetic studies thus indicate a slightly lower degree of oxidation of FeTHT compared to the results from the XPS studies (only $\mathrm{Fe}^{3+}$ is detected by XPS upon exposure of the sample to ambient atmosphere for 7 days). These results demonstrate $\mathrm{O}_{2}$-treatment as an accessible tool for surface-localized chemical oxidation of 2D MOFs.

\section{Resistivity Studies}

The temperature-dependent resistivity of FeTHT was measured using a four-point Van der Pauw geometry. Films of thicknesses ranging from 84(8) nm to 410(41) nm, as determined by AFM studies (Figure S24), were analysed under identical conditions. Ohmic contacts, as demonstrated by linear I-V traces (Figure S25), were made using graphitic carbon adhesive in ambient atmosphere with minimal ( $<10 \mathrm{~min}$ ) air exposure. At $300 \mathrm{~K}$, the bulk resistivity of the $275 \mathrm{~nm}$-thick FeTHT film was determined to be $5.4 \Omega \cdot \mathrm{cm}$, corresponding to a conductivity of 0.2 S/cm (Figures 5 and S26, and Table S7). Cooling from $300 \mathrm{~K}$ to $140 \mathrm{~K}$ results in an increased resistivity, as expected for a semiconducting material where transport is dominated by thermallypopulated carriers (Figures 5 and S26). This is similar to what was observed for films and pressed pellets of CoTHT at elevated temperatures, and is consistent with the report of semiconducting behavior for $\mathrm{Fe}_{3}(\mathrm{THT})_{2}\left(\mathrm{NH}_{4}\right)_{3}$ (Table S8). ${ }^{52,55}$ An Arrhenius fit to the resistivity data recorded between 300 and $200 \mathrm{~K}$ indicates an activation energy for electron hopping between 6 and 13 meV (Figures S27 and S28), which suggests defects, most likely grain boundaries in the 2D sheets, significantly influence the charge transport.

Below $140 \mathrm{~K}$, the FeTHT film exhibits a maximum in the resistivity followed by a change in slope to decrease below 100K (Figures 5 and S26, and Table S7). These observations are qualitatively very similar to the behavior of CoTHT, ${ }^{52}$ and are fully-reversible with no signatures of thermal hysteresis. Given the complex nature of the transition, $T_{\text {metallic }}$ is defined as the lowest transition temperature at which FeTHT becomes metallic $(100 \mathrm{~K})$. No morphological changes are observed by scanning electron microscopy (Figure S29). The measured semiconductor-to-metal transition temperatures ( $T_{\text {metallic}}$ ) for FeTHT do not display any appreciable shifts with film thickness (Figure S26 and Table S7).

To further examine the influence that oxidation has on the conductivity, resistivity measurements were collected on samples exposed to air for various durations. The original 275(28) nm-thick FeTHT film was exposed to the ambient atmosphere, while taking care to maintain the original contacts. After two and a half days of exposure, the resistivity was observed to increase nearly ten-fold, from 5.2 to $47.5 \Omega \cdot \mathrm{cm}$ at $300 \mathrm{~K}$ (Figure 5). Remarkably, this coincides with a signficant increase in the maxima of the resisitivity from 100 to $135 \mathrm{~K}$. Further oxidation over a one month period led to a shift to approximately $300 \mathrm{~K}$ with a corresponding increase in 
resistivity to $735 \Omega \cdot \mathrm{cm}$. By one and a half months, the transition was shifted to room temperature and exhibited a $\rho_{300 \mathrm{~K}}$ of $1,260 \Omega \cdot \mathrm{cm}$. These effects were seen to accelerate when a $310(31) \mathrm{nm}$ thick sample of FeTHT was heated in air at $60{ }^{\circ} \mathrm{C}$ for three days (Figure S30). In contrast, heating the sample for the same duration under helium shows nearly no change in the position of the maxima (Figure S31), which supports the conclusion that the shifts in the transition temperature are related to the oxidation of $\mathrm{Fe}^{2+}$ to $\mathrm{Fe}^{3+}$.

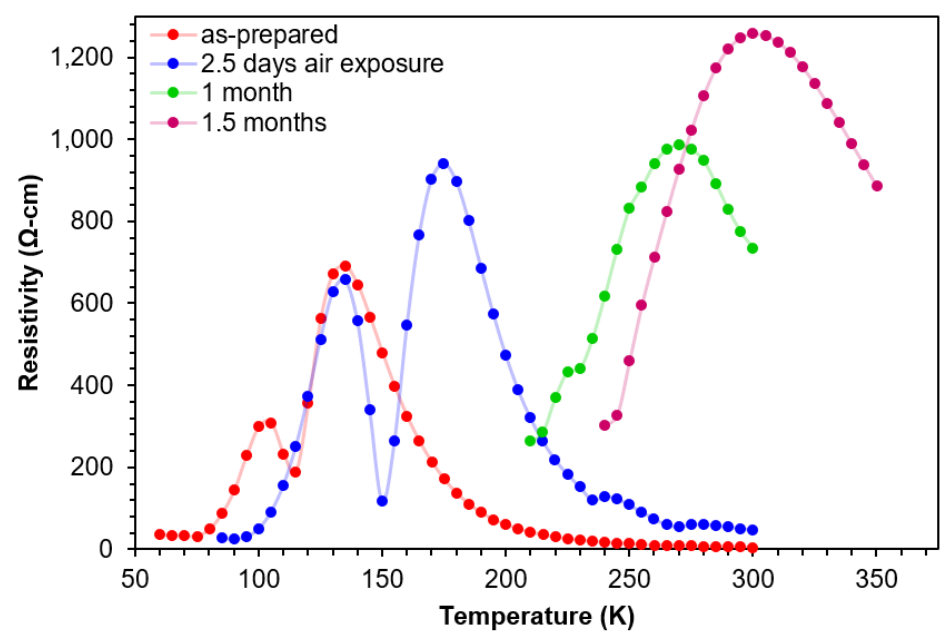

Figure 5. Temperature-dependent resistivity data for FeTHT films with 275(28) nm thickness, asprepared with minimal ( $<10 \mathrm{~min}$ ) air exposure (red) and following exposure to ambient atmosphere for 2.5 days (blue), 1 month (green) and 1.5 months (magenta).

\section{CONCLUSIONS}

We have presented a modified synthesis for the recently-reported iron 2,3,6,7,10,11tripheylenehexathiolate (FeTHT) MOF, generating a material with unusual electronic properties that is isostructural to the previously reported CoTHT. Temperature-dependent resistivity studies reveal a transition from semiconducting to metallic character on cooling, which is analogous to the behaviour observed for CoTHT. This contrasts with previously reported measurements on FeTHT where $\mathrm{NH}_{4}{ }^{+}$was present as a guest species and exhibited purely semiconducting behavior. Interestingly, oxidation of the material on exposure to ambient conditions has a significant effect on the semiconducting-to-metallic transition temperature over time, resulting in a material with metallic-like behavior at room temperature. Through a combination of X-ray photoelectron spectroscopy (XPS) and magnetic susceptibility studies, we have shown that both the metal center and the ligand backbone are susceptible to oxidation. These results indicate the semiconducting-to-metallic transition is strongly influenced by changes in the degree of doping of the material and provides greater insight into the possible origin of this behavior.

\section{ACKNOWLEDGEMENTS}

We are grateful to the University of Southern California (USC) and the USC Women in Science and Engineering for funding. A.J.C. gratefully acknowledges support from the Norma and Jerol Sonosky Fellowship and the USC Wrigley Institute. A.J.N and B.C.M. gratefully acknowledge funding through 
Office of Naval Research Grant No. N00014-15-1-2411. S.A.H. was supported as part of the Multidisciplinary GAANN in Smart Energy Materials, a Graduate Areas of National Need, funded by the U.S. Department of Education, under Award P200A150135. J.M.S. is grateful to the UK Engineering and Physical Sciences Council (Grant No. EP/P007821/1) and to the University of Manchester for the support of a Presidential Fellowship. SEM data were collected at the Center for Electron Microscopy and Microanalysis (CEMMA), USC. Use of the Advanced Photon Source at Argonne National Laboratory was supported by the U. S. Department of Energy, Office of Science, Office of Basic Energy Sciences, under Contract No. DE-AC02-06CH11357. We are grateful to Prof. Mark Thompson for allowing the use of the Agilent 5420 SPM instrument for AFM studies. We thank Sara Smock for assistance with the BET measurements. The computational modelling studies were carried out using the UK Archer HPC facility through the UK Materials Chemistry Consortium, which is funded by the EPSRC (Grant. No. EP/L000202).

Competing Financial Interests. The authors declare no competing financial interests.

Materials \& Correspondence. smarines@usc.edu, and melot@usc.edu.

\section{References}

(1) Furukawa, H.; Cordova, K. E.; O’Keeffe, M.; Yaghi, O. M. The Chemistry and Applications of Metal-Organic Frameworks. Science 2013, 341, 1230444.

(2) Zhou, H.-C.; Long, J. R.; Yaghi, O. M. Introduction to Metal-Organic Frameworks. Chem. Rev. 2012, 112, 673-674.

(3) Allendorf, M. D.; Stavila, V. Crystal engineering, structure-function relationships, and the future of metal-organic frameworks. CrystEngComm 2015, 17, 229-246.

(4) Meek, S. T.; Greathouse, J. A.; Allendorf, M. D. Metal-Organic Frameworks: A Rapidly Growing Class of Versatile Nanoporous Materials. Adv. Mater. 2011, 23, 249-267.

(5) Hendon, C. H.; Rieth, A. J.; Korzyński, M. D.; Dincă, M. Grand Challenges and Future Opportunities for Metal-Organic Frameworks. ACS Centr. Sci. 2017, 3, 554-563.

(6) Liu, G.; Chernikova, V.; Liu, Y.; Zhang, K.; Belmabkhout, Y.; Shekhah, O.; Zhang, C.; Yi, S.; Eddaoudi, M.; Koros, W. J. Mixed matrix formulations with MOF molecular sieving for key energy-intensive separations. Nat. Mater. 2018, 17, 283-289.

(7) Qiu, S.; Xue, M.; Zhu, G. Metal-organic framework membranes: from synthesis to separation application. Chem. Soc. Rev. 2014, 43, 6116-6140.

(8) Schoedel, A.; Ji, Z.; Yaghi, O. M. The role of metal-organic frameworks in a carbonneutral energy cycle. Nat. Energy 2016, 1, 16034.

(9) Zhang, T.; Lin, W. Metal-organic frameworks for artificial photosynthesis and photocatalysis. Chem. Soc. Rev. 2014, 43, 5982-5993.

(10) Yaghi, O. M.; O'Keeffe, M.; Ockwig, N. W.; Chae, H. K.; Eddaoudi, M.; Kim, J. Reticular synthesis and the design of new materials. Nature 2003, 423, 705-714.

(11) Sun, L.; Campbell, M. G.; Dincă, M. Electrically Conductive Porous Metal-Organic Frameworks. Angew. Chem. Int. Ed. 2016, 55, 2-16.

(12) Stassen, I.; Burtch, N.; Talin, A.; Falcaro, P.; Allendorf, M.; Ameloot, R. An updated roadmap for the integration of metal-organic frameworks with electronic devices and chemical sensors. Chem. Soc. Rev. 2017, 46, 3185-3241.

(13) Downes, C. A.; Marinescu, S. C. Electrocatalytic Metal-Organic Frameworks for Energy Applications. ChemSusChem 2017, 10, 4374-4392. 
(14) Hendon, C. H.; Tiana, D.; Walsh, A. Conductive metal-organic frameworks and networks: fact or fantasy? Phys. Chem. Chem. Phys. 2012, 14, 13120-13132.

(15) Allendorf, M. D.; Schwartzberg, A.; Stavila, V.; Talin, A. A. A Roadmap to Implementing Metal-Organic Frameworks in Electronic Devices: Challenges and Critical Directions. Chem. Eur. J. 2011, 17, 11372-11388.

(16) Kung, C.-W.; Otake, K.; Buru, C. T.; Goswami, S.; Cui, Y.; Hupp, J. T.; Spokoyny, A. M.; Farha, O. K. Increased Electrical Conductivity in a Mesoporous Metal-Organic Framework Featuring Metallacarboranes Guests. J. Am. Chem. Soc. 2018, 140, 3871-3875.

(17) Talin, A. A.; Centrone, A.; Ford, A. C.; Foster, M. E.; Stavila, V.; Haney, P.; Kinney, R. A.; Szalai, V.; El Gabaly, F.; Yoon, H. P.; Léonard, F.; Allendorf, M. D. Tunable Electrical Conductivity in Metal-Organic Framework Thin-Film Devices. Science 2014, 343, 66-69.

(18) Mulzer, C. R.; Shen, L.; Bisbey, R. P.; McKone, J. R.; Zhang, N.; Abruña, H. D.; Dichtel, W. R. Superior Charge Storage and Power Density of a Conducting Polymer-Modified Covalent Organic Framework. ACS Centr. Sci. 2016, 2, 667-673.

(19) Kobayashi, Y.; Jacobs, B.; Allendorf, M. D.; Long, J. R. Conductivity, Doping, and Redox Chemistry of a Microporous Dithiolene-Based Metal-Organic Framework. Chem. Mater. 2010, $22,4120-4122$.

(20) Cai, S.-L.; Zhang, Y.-B.; Pun, A. B.; He, B.; Yang, J.; Toma, F. M.; Sharp, I. D.; Yaghi, O. M.; Fan, J.; Zheng, S.-R.; Zhang, W.-G.; Liu, Y. Tunable electrical conductivity in oriented thin films of tetrathiafulvalene-based covalent organic framework. Chem. Sci. 2014, 5, 4693-4700.

(21) Cui, J.; Xu, Z. An electroactive porous network from covalent metal-dithiolene links. Chem. Commun. 2014, 50, 3986-3988.

(22) Murase, R.; Leong, C. F.; D’Alessandro, D. M. Mixed Valency as a Strategy for Achieving Charge Delocalization in Semiconducting and Conducting Framework Materials. Inorg. Chem. 2017, 56, 14373-14382.

(23) Sun, L.; Hendon, C. H.; Park, S. S.; Tulchinsky, Y.; Wan, R.; Wang, F.; Walsh, A.; Dincă, M. Is iron unique in promoting electrical conductivity in MOFs? Chem. Sci. 2017, 8, 4450-4457.

(24) Manumpil, M. A.; Leal-Cervantes, C.; Hudson, M. R.; Brown, C. M.; Karunadasa, H. I. Electronic Conductivity in a Porous Vanadyl Prussian Blue Analogue upon Air Exposure. Inorg. Chem. 2017, 56, 12682-12686.

(25) Xie, L. S.; Sun, L.; Wan, R.; Park, S. S.; DeGayner, J. A.; Hendon, C. H.; Dincă, M. Tunable Mixed-Valence Doping toward Record Electrical Conductivity in a Three-Dimensional Metal-Organic Framework. J. Am. Chem. Soc. 2018, 140, 7411-7414.

(26) Park, J. G.; Aubrey, M. L.; Oktawiec, J.; Chakarawet, K.; Darago, L. E.; Grandjean, F.; Long, G. J.; Long, J. R. Charge Delocalization and Bulk Electronic Conductivity in the Mixed-Valence Metal-Organic Framework Fe(1,2,3-triazolate $)_{2}\left(\mathrm{BF}_{4}\right)_{\mathrm{x}}$. J. Am. Chem. Soc. 2018, 140, 8526-8534.

(27) Narayan, T. C.; Miyakai, T.; Seki, S.; Dincă, M. High Charge Mobility in a Tetrathiafulvalene-Based Microporous Metal-Organic Framework. J. Am. Chem. Soc. 2012, 134, 1293212935.

(28) Park, S. S.; Hontz, E. R.; Sun, L.; Hendon, C. H.; Walsh, A.; Van Voorhis, T.; Dincă, M. Cation-Dependent Intrinsic Electrical Conductivity in Isostructural Tetrathiafulvalene-Based Microporous Metal-Organic Frameworks. J. Am. Chem. Soc. 2015, 137, 1774-1777.

(29) Sun, L.; Miyakai, T.; Seki, S.; Dincă, M. $\mathrm{Mn}_{2}$ (2,5-disulfhydrylbenzene-1,4dicarboxylate): A Microporous Metal-Organic Framework with Infinite (-Mn-S-)œ Chains and High Intrinsic Charge Mobility. J. Am. Chem. Soc. 2013, 135, 8185-8188.

(30) Ko, M.; Mendecki, L.; Mirica, K. A. Conductive two-dimensional metal-organic frameworks as multifunctional materials. Chem. Commun. 2018, 54, 7873-7891.

(31) D'Alessandro, D. M. Exploiting redox activity in metal-organic frameworks: concepts, trends and perspectives. Chem. Commun. 2016, 52, 8957-8971.

(32) Maeda, H.; Sakamoto, R.; Nishihara, H. Coordination Programming of Two-Dimensional Metal Complex Frameworks. Langmuir 2016, 32, 2527-2538. 
(33) Sakamoto, R.; Takada, K.; Pal, T.; Maeda, H.; T., K.; Nishihara, H. Coordination nanosheets (CONASHs): strategies, structures and functions. Chem. Commun. 2017, 53, 5781-5801.

(34) Calbo, J.; Golomb, M. J.; Walsh, A. Redox-active metal-organic frameworks for energy conversion and storage. J. Mater. Chem. A 2019, 7, 16571-16597.

(35) Darago, L. E.; Aubrey, M. L.; Yu, C. J.; Gonzalez, M. I.; Long, J. R. Electronic Conductivity, Ferrimagnetic Ordering, and Reductive Insertion Mediated by Organic Mixed-Valence in a Ferric Semiquinoid Metal-Organic Framework. J. Am. Chem. Soc. 2015, 137, 15703-15711.

(36) Ziebel, M. E.; Darago, L. E.; Long, J. R. Control of Electronic Structure and Conductivity in Two-Dimensional Metal-Semiquinoid Frameworks of Titanium, Vanadium, and Chromium. J. Am. Chem. Soc. 2018, 140, 3040-3051.

(37) Hmadeh, M.; Lu, Z.; Liu, Z.; Gándara, F.; Furukawa, H.; Wan, S.; Augustyn, V.; Chang, R.; Liao, L.; Zhou, F.; Perre, E.; Ozolins, V.; Suenaga, K.; Duan, X.; Dunn, B.; Yamamto, Y.; Terasaki, O.; Yaghi, O. M. New Porous Crystals of Extended Metal-Catecholates. Chem. Mater. 2012, 24, 35113513.

(38) Park, J.; Hinckley, A. C.; Huang, Z.; Feng, D.; Yakovenko, A. A.; Lee, M.; Chen, S.; Zou, X.; Bao, Z. Synthetic Routes for a 2D Semiconductive Copper Hexahydroxybenzene Metal-Organic Framework. J. Am. Chem. Soc. 2018, 140, 14533-14537.

(39) Hoppe, B.; Hindricks, K. D. J.; Warwas, D. P.; Schulze, H. A.; Mohmeyer, A.; Pinkvos, T. J.; Zailskas, S.; Krey, M. R.; Belke, C.; König, S.; Fröba, M.; Haug, R. J.; Behrens, P. Graphene-like metal-organic frameworks: morphology control, optimization of thin film electrical conductivity and fast sensing applications. CrystEng Comm 2018, 20, 6458-6471.

(40) Campbell, M. G.; Liu, S. F.; Swager, T. M.; Dincă, M. Chemiresistive Sensor Arrays from Conductive 2D Metal-Organic Frameworks. J. Am. Chem. Soc. 2015, 137, 13780-13783.

(41) Yao, M.-S.; Lv, X.-J.; Fu, Z.-H.; Li, W.-H.; Deng, W.-H.; Wu, G.-D.; Xu, G. Layer-byLayer Assembled Conductive Metal-Organic Framework Nanofilms for Room-Temperature Chemiresistive Sensing. Angew. Chem. Int. Ed. 2017, 56, 16510-16514.

(42) Rubio-Giménez, V. c.; Galbiati, M.; Castells-Gil, J.; Almora-Barrios, N.; NavarroSánchez, J.; Escorcia-Ariza, G.; Mattera, M.; Arnold, T.; Rawle, J.; Tatay, S.; Coronado, E.; MartíGastaldo, C. Bottom-Up Fabrication of Semiconductive Metal-Organic Framework Ultrathin Films. Adv. Mater. 2018, 30, 1704291.

(43) Ko, M.; Aykanat, A.; Smith, M. K.; Mirica, K. A. Drawing Sensors with Ball-Milled Blends of Metal-Organic Frameworks and Graphite. Sensors 2017, 17, 2192.

(44) Sheberla, D.; Sun, L.; Blood-Forsythe, M. A.; Er, S.; Wade, C. R.; Brozek, C. K.; Aspuru-Guzik, A.; Dincă, M. High Electrical Conductivity in $\mathrm{Ni}_{3}(2,3,6,7,10,11 \text {-hexaiminotriphenylene })_{2}$, a Semiconducting Metal-Organic Graphene Analogue. J. Am. Chem. Soc. 2014, 136, 8859-8862.

(45) Sun, L.; Liao, B.; Sheberla, D.; Kraemer, D.; Zhou, J.; Stach, E. A.; Zakharov, D.; Stavila, V.; Talin, A. A.; Ge, Y.; Allendorf, M. D.; Chen, G.; Léonard, F.; Dincă, M. A Microporous and Naturally Nanostructured Thermoelectric Metal-Organic Framework with Ultralow Thermal Conductivity. Joule 2017, 1, 168-177.

(46) Dou, J.-H.; Sun, L.; Ge, Y.; Li, W.; Hendon, C. H.; Li, J.; Gul, S.; Yano, J.; Stach, E. A.; Dincă, M. Signature of Metallic Behavior in the Metal-Organic Frameworks $\mathrm{M}_{3}$ (hexaiminobenzene) 2 (M = Ni, Cu). J. Am. Chem. Soc. 2017, 139, 13608-13611.

(47) Feng, D.; Lei, T.; Lukatskaya, M. R.; Park, J.; Huang, Z.; Lee, M.; Shaw, L.; Chen, S.; Yakovenko, A. A.; Kulkarni, A.; Xiao, J.; Fredrickson, K.; Tok, J. B.; Zou, X.; Cui, Y.; Bao, Z. Robust and conductive two-dimensional metal- organic frameworks with exceptionally high volumetric and areal capacitance. Nat. Energy 2018, 3, 30-36.

(48) Park, J.; Lee, M.; Feng, D.; Huang, Z.; Hinckley, A. C.; Yakovenko, A.; Zou, X.; Cui, Y.; Bao, Z. Stabilization of Hexaaminobenzene in a 2D Conductive Metal- Organic Framework for High Power Sodium Storage. J. Am. Chem. Soc. 2018, 140, 10315-10323. 
(49) Huang, X.; Sheng, P.; Tu, Z.; Zhang, F.; Wang, J.; Geng, H.; Zou, Y.; Di, C.-a.; Yi, Y.; Sun, Y.; Xu, W.; Zhu, D. A two-dimensional $\pi$-d conjugated coordination polymer with extremely high electrical conductivity and ambipolar transport behaviour. Nat. Commun. 2015, 6, 7408.

(50) Kambe, T.; Sakamoto, R.; Kusamoto, T.; Pal, T.; Fukui, N.; Hoshiko, K.; Shimojima, T.; Wang, Z.; Hirahara, T.; Ishizaka, K.; Hasegawa, S.; Liu, F.; Nishihara, H. Redox Control and High Conductivity of Nickel Bis(dithiolene) Complex $\pi$-Nanosheet: A Potential Organic Two-Dimensional Topological Insulator. J. Am. Chem. Soc. 2014, 136, 14357-14360.

(51) Sun, X.; Wu, K.-H.; Sakamoto, R.; Kusamoto, T.; Maeda, H.; Ni, X.; Jiang, W.; Liu, F.; Sasaki, S.; Masunaga, H.; Nishihara, H. Bis(aminothiolato)nickel nanosheet as a redox switch for conductivity and an electrocatalyst for the hydrogen evolution reaction. Chem. Sci. 2017, 8, 8078-8085.

(52) Clough, A. J.; Skelton, J. M.; Downes, C. A.; de la Rosa, A. A.; Yoo, J. W.; Walsh, A.; Melot, B. C.; Marinescu, S. C. Metallic Conductivity in a Two-Dimensional Cobalt Dithiolene MetalOrganic Framework. J. Am. Chem. Soc. 2017, 139, 10863-10867.

(53) Takaishi, S.; Hosoda, M.; Kajiwara, T.; Miyasaka, H.; Yamashita, M.; Nakanishi, Y.; Kitagawa, Y.; Yamaguchi, K.; Kobayashi, A.; Kitagawa, H. Electroconductive Porous Coordination Polymer $\mathrm{Cu}\left[\mathrm{Cu}(\mathrm{pdt})_{2}\right]$ Composed of Donor and Acceptor Building Units. Inorg. Chem. 2009, 48, 90489050.

(54) Kambe, T.; Sakamoto, R.; Hoshiko, K.; Takada, K.; Miyachi, M.; Ryu, J.-H.; Sasaki, S.; Kim, J.; Nakazato, K.; Takata, M.; Nishihara, H. $\pi$-Conjugated Nickel Bis(dithiolene) Complex Nanosheet. J. Am. Chem. Soc. 2013, 135, 2462-2465.

(55) Dong, R.; Han, P.; Arora, H.; Ballabio, M.; Karakus, M.; Zhang, Z.; Shekhar, C.; Adler, P.; Petkov, P. S.; Erbe, A.; Mannsfeld, S. C. B.; Felser, C.; Heine, T.; Bonn, M.; Feng, X.; Cánovas, E. High-mobility band-like charge transport in a semiconducting two-dimensional metal-organic framework. Nat. Mater. 2018, 17, 1027-1032.

(56) Sun, X.; Wu, K.-H.; Sakamoto, R.; Kusamoto, T.; Maeda, H.; Nishihara, H. Conducting $\pi$-Conjugated Bis(iminothiolato)nickel Nanosheet. Chem. Lett. 2017, 46, 1072-1075.

(57) Dong, R.; Zhang, Z.; Tranca, D. C.; Zhou, S.; Wang, M.; Adler, P.; Liao, Z.; Liu, F.; Sun, Y.; Shi, W.; Zhang, Z.; Zschech, E.; Mannsfeld, S. C. B.; Felser, C.; Feng, X. A coronene-based semiconducting two- dimensional metal-organic framework with ferromagnetic behavior. Nat. Commun. 2018, 9, 2637.

(58) Huang, X.; Li, H.; Tu, Z.; Liu, L.; Wu, X.; Chen, J.; Liang, Y.; Zou, Y.; Yi, Y.; Sun, J.; $\mathrm{Xu}, \mathrm{W}$.; Zhu, D. Highly Conducting Neutral Coordination Polymer with Infinite Two- Dimensional Silver-Sulfur Networks. J. Am. Chem. Soc. 2018, 140, 15153-15156.

(59) Chen, I.-F.; Lu, C.-F.; Su, W.-F. Highly Conductive 2D Metal-Organic Framework Thin Film Fabricated by Liquid-Liquid Interfacial Reaction Using One-Pot- Synthesized Benzenehexathiol. Langmuir 2018, 34, 15754-15762.

(60) Pal, T.; Doi, S.; Maeda, H.; Wada, K.; Tan, C. M.; Fukui, N.; Sakamoto, R.; Tsuneyuki, S.; Sasaki, S.; Nishihara, H. Interfacial Transmetallation Synthesis of Platinadithiolene Nanosheet as a Potential 2D Topological Insulator. Chem. Sci. 2019, 10, 5218-5225.

(61) Huang, X.; Zhang, S.; Liu, L.; Yu, L.; Chen, G.; Xu, W.; Zhu, D. Superconductivity in a Copper(II)-Based Coordination Polymer with Perfect Kagome Structure. Angew. Chem. Int. Ed. 2018, 57, 146-150.

(62) Foster, M. E.; Sohlberg, K.; Allendorf, M. D.; Talin, A. A. Unraveling the Semiconducting/Metallic Discrepancy in $\mathrm{Ni}_{3}(\mathrm{HITP})_{2}$. J. Phys. Chem. Lett. 2018, 9, 481-486.

(63) Chen, S.; Dai, J.; Zeng, X. C. Metal-organic Kagome lattices $M_{3}(2,3,6,7,10,11$ hexaiminotriphenylene $)_{2}(\mathrm{M}=\mathrm{Ni}$ and $\mathrm{Cu})$ : from semiconducting to metallic by metal substitution. Phys. Chem. Chem. Phys. 2015, 17, 5954-5958.

(64) Foster, M. E.; Sohlberg, K.; Spataru, C. D.; Allendorf, M. D. Proposed Modification of the Graphene Analogue $\mathrm{Ni}_{3}(\mathrm{HITP})_{2}$ To Yield a Semiconducting Material. J. Phys. Chem. C 2016, 120, 15001-15008. 
(65) Li, S.; Lü, T.-Y.; Zheng, J.-C.; Yang, S.-W.; Wang, J.-S.; Wu, G. Origin of metallicity in 2D multilayer nickel bis(dithiolene) sheets. 2D Mater. 2018, 5, 035027.

(66) Sproules, S.; Wieghardt, K. Dithiolene radicals: Sulfur K-edge X-ray absorption spectroscopy and Harry's intuition. Coord. Chem. Rev. 2011, 255, 837-860.

(67) Sproules, S.; Wieghardt, K. o-Dithiolene and o-aminothiolate chemistry of iron: Synthesis, structure and reactivity. Coord. Chem. Rev. 2010, 254, 1358-1382.

(68) Eisenberg, R.; Gray, H. B. Noninnocence in Metal Complexes: A Dithiolene Dawn. Inorg. Chem. 2011, 50, 9741-9751.

(69) Clough, A. J.; Yoo, J. W.; Mecklenburg, M. H.; Marinescu, S. C. Two-Dimensional Metal-Organic Surfaces for Efficient Hydrogen Evolution from Water. J. Am. Chem. Soc. 2015, 137, $118-121$.

(70) Grosvenor, A. P.; Kobe, B. A.; Biesinger, M. C.; McIntyre, N. S. Investigation of multiplet splitting of Fe 2p XPS spectra and bonding in iron compounds. Surf. Interface Anal. 2004, 36, 1564-1574.

(71) Fantauzzi, M.; Elsener, B.; Atzei, D.; Rigoldi, A.; Rossi, A. Exploiting XPS for the identification of sulfides and polysulfides. RSC Adv. 2015, 5, 75953-75963

(72) Castner, D. G.; Hinds, K.; Grainger, D. W. X-ray Photoelectron Spectroscopy Sulfur 2p Study of Organic Thiol and Disulfide Binding Interactions with Gold Surfaces. Langmuir 1996, 12, 50835086

(73) Castillo, O.; Delgado, E.; Goméez-García, C. J.; Hernańdez, D.; Hernańdez, E.; Herrasti, P.; Martín, A.; Zamora, F. i. Comparative Studies of Oxidation Processes on Group 10 Metals Dithiolene Derivatives in the Formation of Coordination Polymers. Cryst. Growth Des. 2018, 18, 2486-2494.

(74) D'Alessandro, D. M.; Kanga, J. R. R.; Caddy, J. S. Towards Conducting Metal-Organic Frameworks. Aust. J. Chem. 2011, 64, 718-722.

(75) Aubrey, M. L.; Wiers, B. M.; Andrews, S. C.; Sakurai, T.; Reyes-Lillo, S. E.; Hamed, S. M.; Yu, C.-J.; Darago, L. E.; Mason, J. A.; Baeg, J.-O.; Grandjean, F.; Long, G. J.; Seki, S.; Neaton, J. B.; Yang, P.; Long, J. R. Electron delocalization and charge mobility as a function of reduction in a metalorganic framework. Nat. Mater. 2018, 17, 625-632.

TOC graphic:

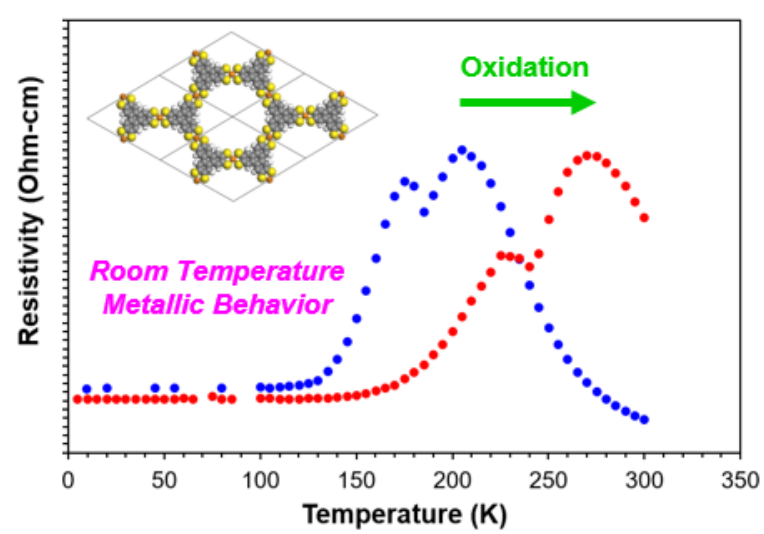




\section{Supporting Information}

Experimental methods, synthetic procedures, computational methods and results, elemental analysis, SEM images, BET results, PXRD data, XPS data, magnetism and conductivity data, and corresponding tabulated results (PDF).

This material is available free of charge via the Internet at http://pubs.acs.org. 


\title{
Room Temperature Metallic Conductivity in a Metal- Organic Framework Induced by Oxidation
}

\author{
Andrew J. Clough, ${ }^{\mathrm{a}}$ Nicholas M. Orchanian, ${ }^{\mathrm{a}}$ Jonathan M. Skelton, ${ }^{\mathrm{b}}$ Abbey J. Neer, ${ }^{\mathrm{a}}$ \\ Sebastian A. Howard, ${ }^{\mathrm{c}}$ Courtney A. Downes, ${ }^{\mathrm{a}}$ Louis F. J. Piper, ${ }^{\mathrm{c}, \mathrm{d}}$ Aron Walsh, ${ }^{\mathrm{e}, \mathrm{f}}$ Brent C. \\ Melot, ${ }^{\text {* }}$ and Smaranda C. Marinescu ${ }^{\mathrm{a} *}$
}

\begin{abstract}
aDepartment of Chemistry, University of Southern California, Los Angeles, California 90089, United States
bDepartment of Chemistry, University of Bath, Bath BA2 7AY, United Kingdom

'Department of Physics, Applied Physics and Astronomy, Binghamton University, Binghamton, New York 13902, United States

${ }^{\mathrm{d}}$ Materials Science \& Engineering, Binghamton University, Binghamton, New York 13902, United States

'Department of Materials, Imperial College London, London SW7 2AZ, United Kingdom

fDepartment of Materials Science and Engineering, Yonsei University, Seoul 03722, Korea
\end{abstract}

*E-mail: smarines@usc.edu; melot@usc.edu.

\section{General}

All manipulations of air and moisture sensitive materials were conducted under a nitrogen atmosphere in a Vacuum Atmospheres drybox or on a dual manifold Schlenk line. The glassware was oven-dried prior to use. Acetonitrile and dichloromethane were degassed with nitrogen and passed through activated alumina columns and stored over $4 \AA$ Linde-type molecular sieves. Ethyl acetate, water, and ethanol were placed under vacuum and refilled with nitrogen $(10 \times)$. Deuterated solvents were dried over $4 \AA$ Linde-type molecular sieves prior to use. Elemental analyses were performed by Robertson Microlit Laboratories, 1705 U.S. Highway 46, Suite 1D, Ledgewood, New Jersey, 07852. All the chemical regents were purchased from commercial vendors and used without further purification. The ligand 2,3,6,7,10,11-triphenylene-hexathiol ${ }^{1}$ (THT) was prepared according to the reported procedures. Water was deionized with the Millipore Milli-Q Synergy system $(18.2 \mathrm{M} \Omega \cdot \mathrm{cm}$ resistivity). All other chemical regents were purchased from commercial vendors and used without further purification.

\section{Synthesis of FeTHT}

The iron triphenylene-2,3,6,7,10,11-hexathiolate framework, FeTHT, was prepared according to the reported procedure for the analogous cobalt framework. ${ }^{2,3}$ A $120 \mathrm{~mL}$ glass jar was charged with an aqueous solution of $\mathrm{FeCl}_{2} \cdot 4 \mathrm{H}_{2} \mathrm{O}$ (40 mg, $0.21 \mathrm{mmol}, 5 \mathrm{mM}, 40 \mathrm{~mL}$ volume). Separately, a suspension of triphenylene-2,3,6,7,10,11-hexathiol (THT) (2 mg, $0.005 \mathrm{mmol}$ ) in N-Methyl-2pyrrolidone (NMP) $(0.2 \mathrm{~mL})$ was diluted with ethyl acetate until the total volume of the suspension reached $20 \mathrm{~mL}$, sealed, and briefly sonicated to form an uniform suspension. Ethyl acetate $(40 \mathrm{~mL})$ was gently added to the aqueous solution of iron(II) chloride to create a liquidliquid interface. The suspension of THT and NMP was then gently added to the ethyl acetate 
layer and the jar was sealed and allowed to stand. A black film appeared at the liquid-liquid interface over 5 days. The film was deposited onto glass supports by pulling the substrate through the film. The deposited films were subsequently washed with water and allowed to evaporate to dryness. Alternatively, the black solid of FeTHT was collected by filtration and washed with water and methanol for bulk powder analyses.

FeTHT powders or films were oxidized by placing them in an oven at $60{ }^{\circ} \mathrm{C}$ for 3 days. Anal. Calcd for $\mathrm{Fe}_{3}\left(\mathrm{THT}_{\mathrm{ox}}\right)_{2} \bullet 3 \mathrm{MeOH} \bullet 12 \mathrm{H}_{2} \mathrm{O}\left(\mathrm{Fe}_{3} \mathrm{C}_{45} \mathrm{H}_{48} \mathrm{~S}_{12} \mathrm{O}_{44}\right)$, where THTox $=\mathrm{C}_{18} \mathrm{H}_{6} \mathrm{O}_{9} \mathrm{~S}_{6}$ : C, 32.38; H, 2.62; Fe, 9.08; S, 23.05. Found: C, 32.89; H, 2.86; Fe, 9.28; S, 23.53.

Powder X-ray diffraction (PXRD) studies were performed on a Rigaku Ultima IV X-Ray diffractometer in reflectance parallel beam/parallel slit alignment geometry. The measurement employed $\mathrm{Cu} \mathrm{K \alpha}$ line focused radiation at $1760 \mathrm{~W}(40 \mathrm{kV}, 44 \mathrm{~mA})$ power and a Ge crystal detector fitted with a $2 \mathrm{~mm}$ radiation entrance slit. Samples were mounted on zero-background sample holders and were observed using a $0.08^{\circ} 2 \theta$ step scan from $2.0-40.0^{\circ}$ with an exposure time of $0.4 \mathrm{~s}$ per step. No peaks could be resolved from the baseline for $2 \theta>35^{\circ}$.

High resolution synchrotron powder X-ray diffraction data was collected using the 11BM beamline mail-in program at the Advanced Photon Source (APS), Argonne National Laboratory, with an average wavelength of $0.414576 \AA$. Discrete detectors covering an angular range from 0.5 to $30^{\circ} 2 \theta$ are scanned over a $34^{\circ} 2 \theta$ range, with data points collected every $0.001^{\circ}$ $2 \theta$ and scan speed of $0.01 \%$. An Oxford Cryosystems Cryostream Plus device allowed for sample temperatures to be controlled over a range of 100-340 K.

\section{Gas Sorption Measurements}

Brunauer-Emmett-Teller (BET) measurements were performed on a Nova 2200e surface area and pore size analyzer (Quantachrome Instruments, Inc.). Samples were degassed for 3 days at $60{ }^{\circ} \mathrm{C}$ in vacuo prior to measurements.

\section{Modeling}

Molecular modeling of FeTHT was carried out using the Materials Studio (version 8.0) suite of programs by Accelrys. The molecular fragment used to generate the model is shown in Figure $\mathrm{S} 1$. The unit cell was constructed starting with a primitive hexagonal unit cell with space group P6/mmm using cell parameters $a=b=22.52 \AA$ and $c=3.3 \AA$. The structure was optimized with Materials Studio Forcite calculations using geometry optimization and universal forcefield methods. The MS Reflex module was used to calculate the expected PXRD patterns. Line broadening for crystallite size was not calculated. Comparison of the simulated and experimental PXRD patterns verified the simulated structure.

\section{Scanning Electron Microscopy (SEM)}

SEM images of FeTHT on glass substrates were collected using a JEOL-7001F or FEI Nova NanoSEM 450 operating at 10 or $15 \mathrm{kV}$ with $5 \mathrm{nA}$ of probe current.

\section{Conductivity Measurements}

FeTHT films were deposited onto glass supports by pulling the substrate (glass) through the FeTHT film formed at the liquid-liquid interface, as described previously. The deposited films were subsequently washed with water and allowed to evaporate to dryness. Conductivity measurements were performed using a custom set up integrated into a 14T Quantum Design 
Dynacool Physical Properties Measurement System. A Keithley 6220 Precision Current Source (excitation currents of 1-50 nA) was used to trigger and control a Keithley 2182A nanovoltmeter. In order to minimize errors associated with contact resistance and drift voltages, a Keithley 2172 matrix switch equipped with a Keithley 6536 Hall effect card was used to alternate the direction of the applied current. Because of difficulty associated with preparing samples with uniform dimensions, all measurements were performed in a four-point probe Van der Pauw geometry. Copper wire contacts were attached to the films using conductive carbon paint and soldered onto a Quantum Design puck with resistivity option. All measurements were performed under a reduced pressure of $\sim 10$ torr.

\section{Magnetic Studies}

Temperature-dependent susceptibility measurements of pristine FeTHT show a paramagnetic response down to $2 \mathrm{~K}$, as illustrated in Figure S23a. A fit of the high temperature magnetic susceptibility $(175-275 \mathrm{~K})$ to the Curie-Weiss law $\chi=\mathrm{C} /\left(\mathrm{T}-\Theta_{\mathrm{cw}}\right)$ yields an effective paramagnetic moment, $\mu_{\mathrm{eff}}=2.20 \mu_{\mathrm{B}}$ per formula unit and a Curie-Weiss temperature $\Theta_{\mathrm{cw}}$ of 0.60 $\mathrm{K}$. The fit, shown in Figure S23b, shows a slight positive deviation corresponding to the positive $\Theta_{\mathrm{cw}}$ of $0.60 \mathrm{~K}$, which indicates that the dominant exchange interaction among the $\mathrm{Fe}$ ions is slightly ferromagnetic. During magnetization, the sample does not saturate up to $14 \mathrm{~T}$ (Figure S23c), which is characteristic of a paramagnetic material.

\section{Atomic Force Microscopy (AFM)}

FeTHT films were deposited onto glass supports by pulling the substrate (glass) through the FeTHT film formed at the liquid-liquid interface, as described previously. The deposited films were subsequently washed with water and allowed to evaporate to dryness. AFM topography images were collected in tapping mode using an Agilent 5420 SPM instrument operating in tapping mode. The probe tips were Tap300-G Silicon AFM probes (resonant frequency $300 \mathrm{kHz}$, force constant $40 \mathrm{~N} / \mathrm{m}$ ) purchased from Budgetsensors.com and aligned prior to use. Images were collected with a scan rate of 0.1 lines per second and over an area of $40 \mu \mathrm{m}$. All samples were imaged under one atmosphere of air at room temperature.

\section{X-Ray Photoelectron Spectroscopy (XPS)}

Care was taken to reduce additional air exposure of the FeTHT samples by mounting in a glovebox with an argon environment and transferring via sealed vacuum suitcase to a lab-based XPS with a monochromatic Al-Ka source and hemispherical analyzer located at the Analytical and Diagnostics Laboratory at Binghamton University. Measurements were performed at room temperature with a pass energy of $23.5 \mathrm{eV}$, corresponding to an instrumental resolution of 0.51 $\mathrm{eV}$, determined from analyzing the Fermi edge and $\mathrm{Au} 4 \mathrm{f}_{7 / 2}$ peak of gold foil references. Lorentzian broadening of Fe $2 \mathrm{p}$ multiplet peaks, necessary to fit Voight profiles in peak fitting, was approximated from the instrumental resolution and $\mathrm{FWHM}$ of $\mathrm{Fe}_{3} \mathrm{O}_{4}$ peaks. ${ }^{4}$ Energy calibration of all core regions were made to $\mathrm{Au} 4 \mathrm{f}_{7 / 2}$ peak $(84.0 \mathrm{eV})$ of gold foil references. 


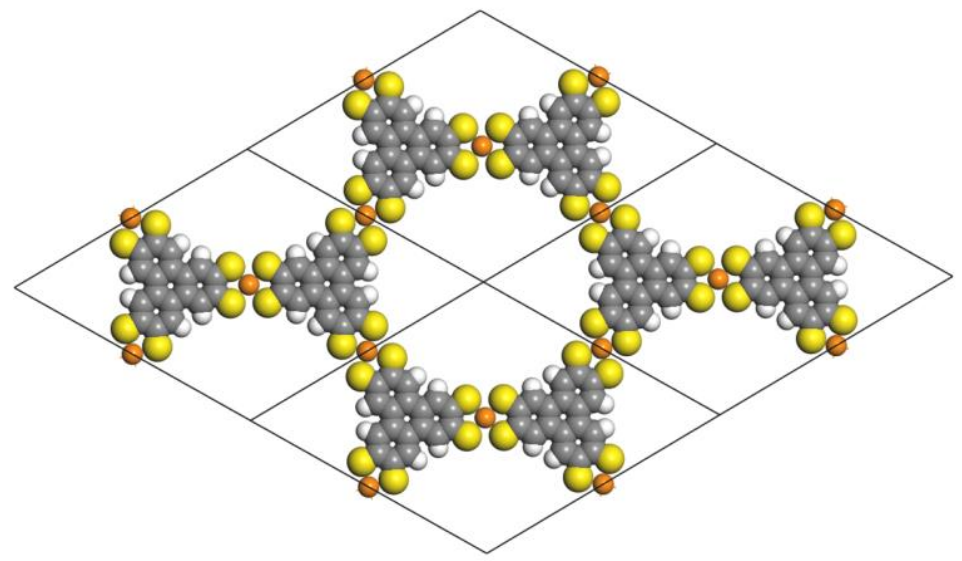

Figure S1. Spacefilling model of the fragment used to generate the unit cell of FeTHT.
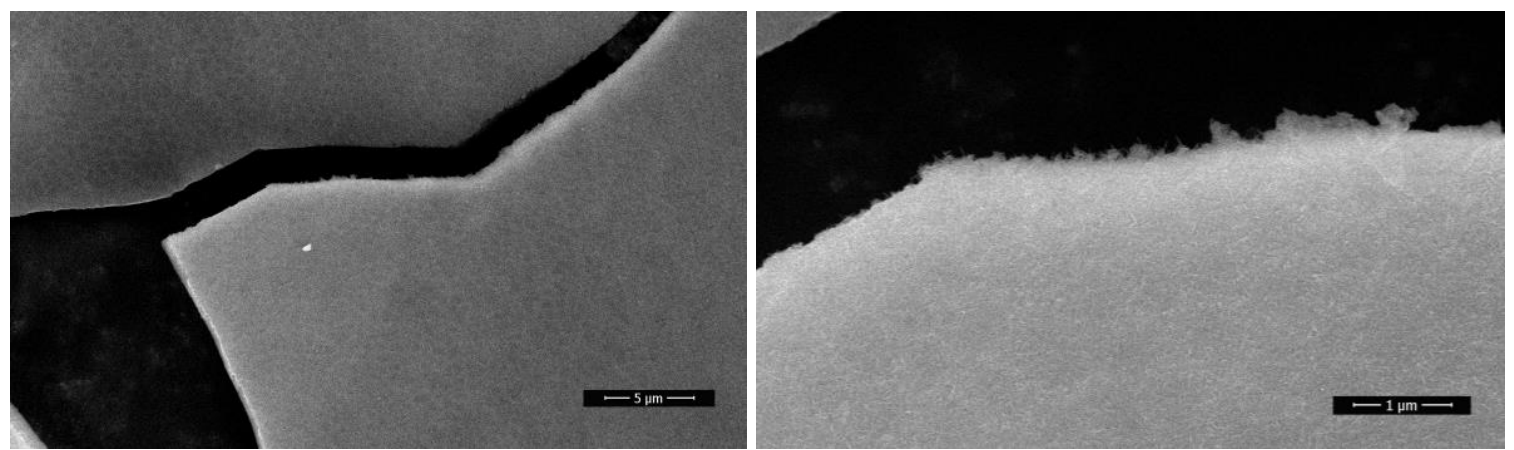

Figure S2. SEM images of FeTHT.

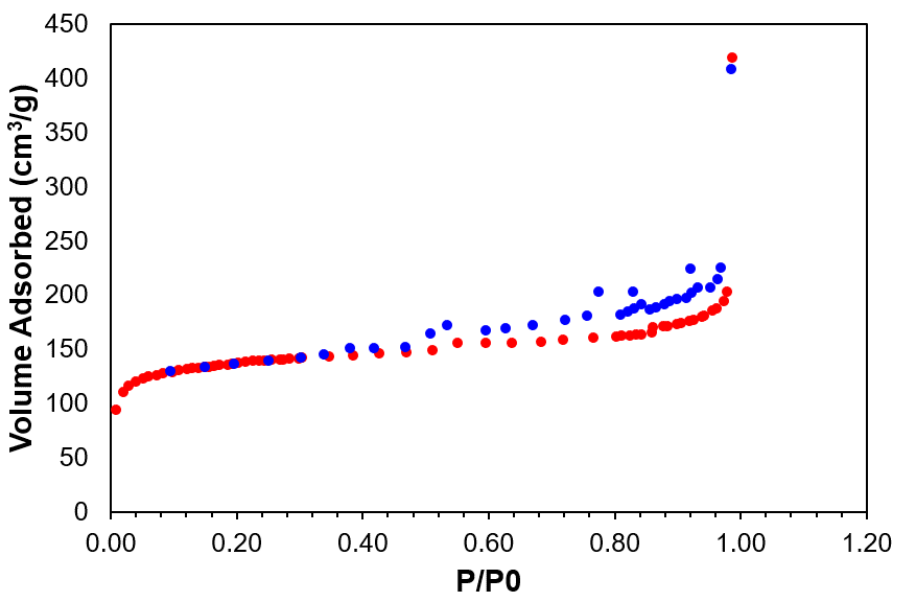

Figure S3. Nitrogen sorption isotherms performed on the as-prepared FeTHT at $77 \mathrm{~K}$ reveal a BET surface area of $441 \mathrm{~m}^{2} / \mathrm{g}$. 


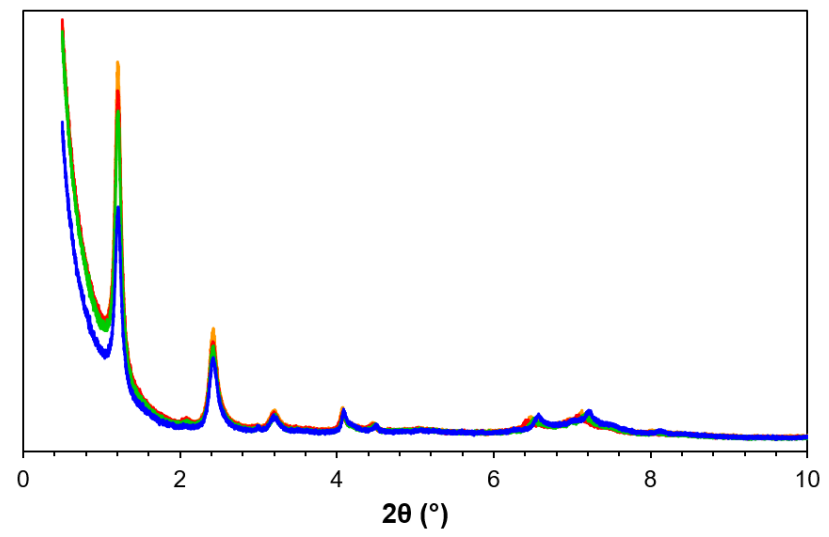

Figure S4. Overlay of the variable temperature PXRD patterns of an oxidized FeTHT sample exposed to air for 3 days at $60{ }^{\circ} \mathrm{C}$. Data was collected at 100 (blue), 200 (green), 295 (orange), and 340 (red) $\mathrm{K}$.
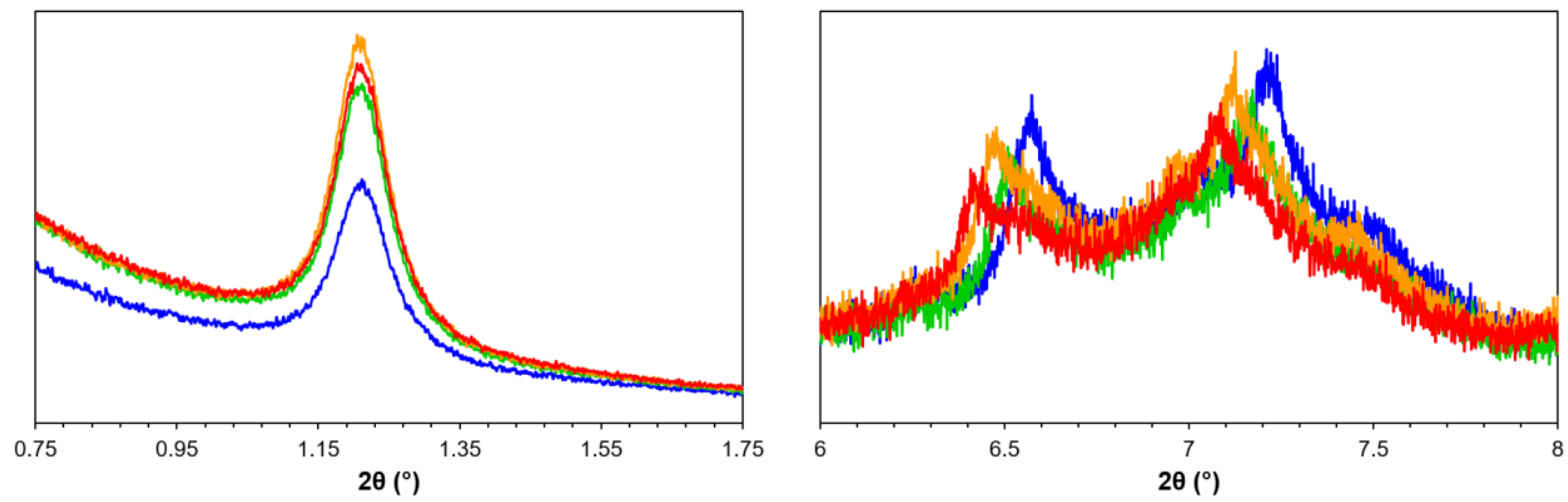

Figure S5. Overlay of the variable temperature PXRD patterns of an oxidized FeTHT sample exposed to air for 3 days at $60{ }^{\circ} \mathrm{C}$, focusing on the [100] (left) and [001] (right) reflections. Data was collected at 100 (blue), 200 (green), 295 (orange), and 340 (red) K.

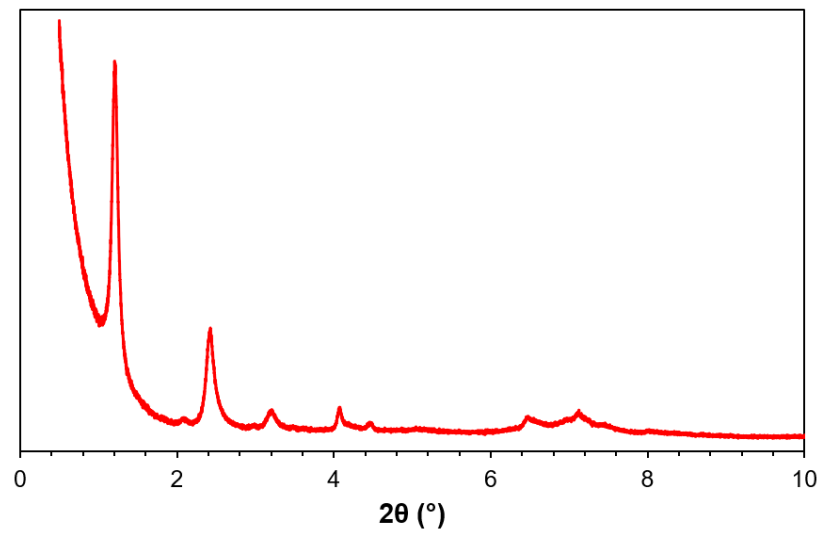

Figure S6. PXRD pattern of an oxidized FeTHT sample exposed to air for 3 days at $60{ }^{\circ} \mathrm{C}$. Data was collected at $295 \mathrm{~K}$. 


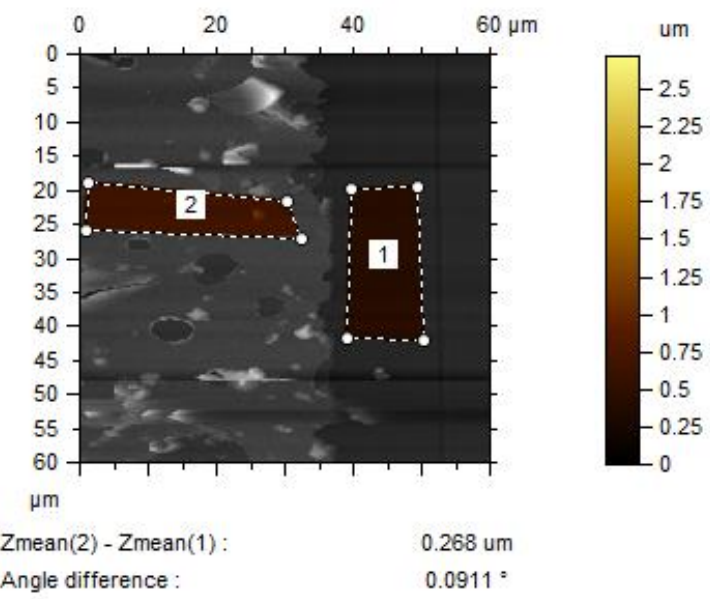

Figure S7. Atomic Force Microscopy (AFM) studies of an oxidized FeTHT sample exposed to air for 3 days at $60{ }^{\circ} \mathrm{C}$. The measured film thickness is $275(28) \mathrm{nm}$.

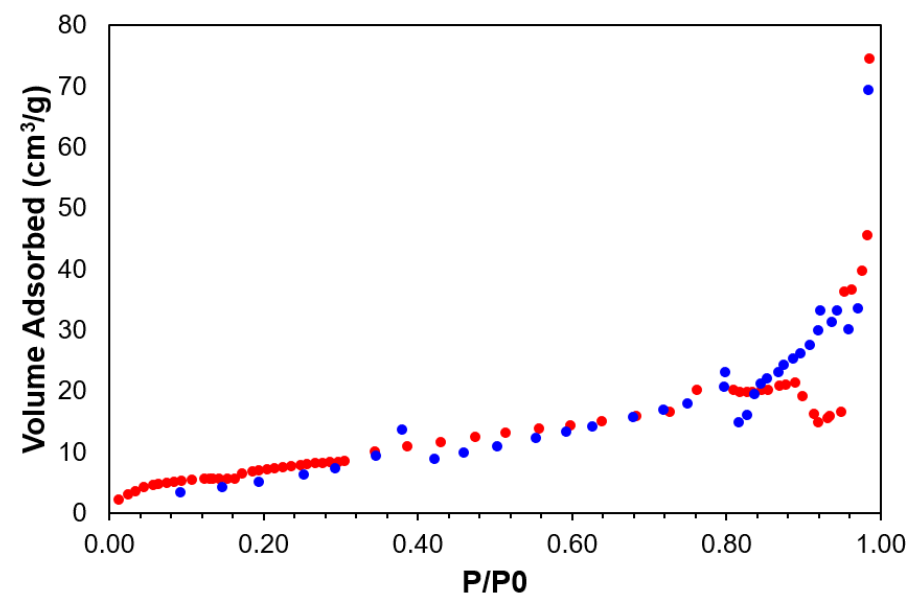

Figure S8. Nitrogen isotherms performed on an oxidized FeTHT sample exposed to air for 3 days at $60{ }^{\circ} \mathrm{C}$. Data collected at $77 \mathrm{~K}$ after air-exposure reveal a BET surface area of $27.6 \mathrm{~m}^{2} / \mathrm{g}$ (red: adsorption, blue: desorption).

\section{Computational Modelling}

Periodic density-functional theory (DFT) calculations on the FeTHT framework were carried out within the pseudopotential plane-wave formalism implemented in the Vienna $A b$ initio Simulation Package (VASP) code $^{5}$

Electron exchange and correlation were modelled with the PBEsol functional ${ }^{6}$ with the DFT-D3 dispersion correction ${ }^{7}$ (i.e. PBEsol-D3). A subset of calculations were repeated with a Hubbard correction of $U_{\mathrm{eff}}=5 \mathrm{eV}$ applied to the Fe d states using the Dudarev method. ${ }^{8}$ 
The ion cores were modelled using projector augmented-wave (PAW) pseudopotentials ${ }^{9,10}$ with the $\mathrm{H} 1 \mathrm{~s}, \mathrm{C} 2 \mathrm{~s} / 2 \mathrm{p}, \mathrm{S} 3 \mathrm{~s} / 3 \mathrm{p}$ and $\mathrm{Fe} 4 \mathrm{~s}, 3 \mathrm{~d}$ and $3 \mathrm{p}$ electrons included in the valence shells. Based on our previous studies of the analogous Co framework, ${ }^{3}$ an $800 \mathrm{eV}$ kinetic-energy cutoff was employed for the plane-wave basis and the electronic wavefunctions were modelled using a $\Gamma$-centred Monkhorst-Pack $k$-point mesh $^{11}$ with $1 \times 1 \times 5$ subdivisions, reduced appropriately for supercell calculations. A Gaussian smearing of $0.01 \mathrm{eV}$ was used to determine partial band occupations.

Tolerances of $10^{-8} \mathrm{eV}$ and $10^{-2} \mathrm{eV} \AA^{-1}$ were applied to the total energy and forces during minimisation of the electronic wavefunctions and geometry optimisations, respectively. The precision of the charge-density grids was set to avoid aliasing errors and the PAW projection was performed in real space.

The electronic density-of-states (DoS) curves were evaluated from a single-point calculation with an increased $k$-point density of $2 \times 2 \times 15$ subdivisions and a larger smearing width of $0.05 \mathrm{eV}$. The charge density from these calculations was then used to model band dispersions by calculating the eigenvalues on strings of $k$-points along high-symmetry directions in the Brillouin zone non self-consistently.

\section{Equilibrium Geometry and Magnetic Structure}

To identify the most energetically-favourable magnetic configuration, we made a starting assumption of a 50/50 mixture of $\mathrm{Fe}^{2+}$ and $\mathrm{Fe}^{3+}$ ions in a square-planar crystal field with zero and one unpaired electron, respectively. A 50/50 mixture of charge states is not compatible with the three $\mathrm{Fe}$ ions in the crystallographic primitive unit cell of the FeTHT framework, so we constructed trial magnetic unit cells based on $1 \times 1 \times 2$ and $2 \times 2 \times 1$ expansions of the primitive cell with $6 / 12 \mathrm{Fe}$ ions and enumerated all symmetry-inequivalent arrangements of the $\mathrm{Fe}^{2+}$ and $\mathrm{Fe}^{3+}$ ions using the Transformer code, ${ }^{12}$ yielding in a total of three and 30 initial configurations in the two cells, respectively. A single-point energy calculation was then carried out on each model, with the magnetic moments allowed to relax during the optimisation of the electronic wavefunctions. We note that this is a more systematic approach than that taken in our previous study on the cobalt analogue (CoTHT). ${ }^{3}$

All 33 initial models relaxed to configurations with magnetic moments around $\pm 2 \mathrm{BM}$ per Fe ion, consistent with all the ions adopting the $\mathrm{Fe}^{3+}$ oxidation state. The calculations on the $1 \times 1 \times 2$ supercell produced one ferromagnetic and one frustrated antiferromagnetic configuration with total moments of 6.3 and 2.0 BM per formula unit (i.e. per single unit cell), respectively. The latter configuration is calculated to be $20 \mathrm{meV}$ per Fe ion lower in energy than the fully ferromagnetic arrangement and is based on chains of Fe ions with parallel spin along the $c$ axis and a mix of ferromagnetic and antiferromagnetic coupling between chains within the layers. A configuration constrained to have ferromagnetic interlayer and antiferromagnetic intralayer coupling was found to be $\sim 0.1 \mathrm{eV}$ per ion higher in energy than the fully ferromagnetic configuration, indicating a strong preference for ferromagnetic coupling between layers.

The majority of the $302 \times 2 \times 1$ models relaxed to antiferromagnetic ground states with an equal number of both spin states, but a small number adopted configurations with two and four excess spins and net magnetic moments of 1.1 and 2.2 BM per formula unit, respectively. The 
energetic differences were on the order of $1-2 \mathrm{meV}$ per $\mathrm{Fe}$ ion, suggesting weak intralayer coupling.

Three of the initial screened configurations were selected for full geometry optimisation, viz. the frustrated antiferromagnetic $1 \times 1 \times 2$ expansion (Model 1), the antiferromagnetic $2 \times 2 \times 1$ expansion (Model 2), and the $2 \times 2 \times 1$ expansion with the larger magnetic moment of $2.2 \mathrm{BM}$ per formula unit (Model 3). The optimised lattice parameters, total magnetic moments and total energies are compared in Table S1.

\begin{tabular}{lcccc}
\hline & Expt. & Model 1 & Model 2 & Model 3 \\
\hline Supercell & - & $1 \times 1 \times 2$ & $2 \times 2 \times 1$ & $2 \times 2 \times 1$ \\
\hline$a[\AA]$ & 22.52 & 23.23 & 23.22 & 23.22 \\
\hline$c[\AA]$ & 3.3 & 3.299 & 3.256 & 3.335 \\
\hline$c / a$ & 0.147 & 0.142 & 0.143 & 0.144 \\
\hline$V\left[\AA^{3}\right]$ & 1449 & 1541 & 1553 & 1557 \\
$M[\mathrm{BM}]$ & 1.87 & 3.04 & -0.03 & 2.21 \\
\hline$E_{0}[\mathrm{eV}$ per Fe ion] & - & -155.24 & -155.30 & -155.30 \\
\hline
\end{tabular}

Table S1. Optimised lattice parameters, total magnetic moments and total energies of three trial magnetic supercells of the FeTHT framework. The lattice parameters and magnetic moments are given with respect to the crystallographic unit cell (i.e. containing three $\mathrm{Fe}$ ions), and the total energies are given per Fe ion. The experimental values are shown in the second column for comparison.

Given the weak intralayer coupling, the $1 \times 1 \times 2$ and net magnetic $2 \times 2 \times 1$ configurations (Models 1 and 3) should be roughly equivalent, and the differences in Table 1 provide an estimate of how much variation can be expected due to differences in the choice of unit cell and associated technical parameters such as the $k$-point sampling. With this in mind, the only major difference between all three models is the magnetic moment. Since Models 1 and 3 can be represented to some level of approximation with a single crystallographic unit cell, which is much more computationally tractable than either supercell, we opted to do this in our production calculations. This also has the advantage of making the calculations directly comparable to those in our previous work on the CoTHT analogue. ${ }^{3}$

Using the single-cell configuration, we obtained optimised lattice parameters of $a=23.21$ and $c=3.334 \AA$, which are a good match to the experimental measurements of 22.52 and $3.3 \AA$ respectively. The calculated spin density (Figure S9) shows that the unpaired electrons are 
localised to the Fe d orbitals, as expected, with a small amount of density on the coordinating $\mathrm{S}$ atoms and very little on the ligand $\pi$ system.

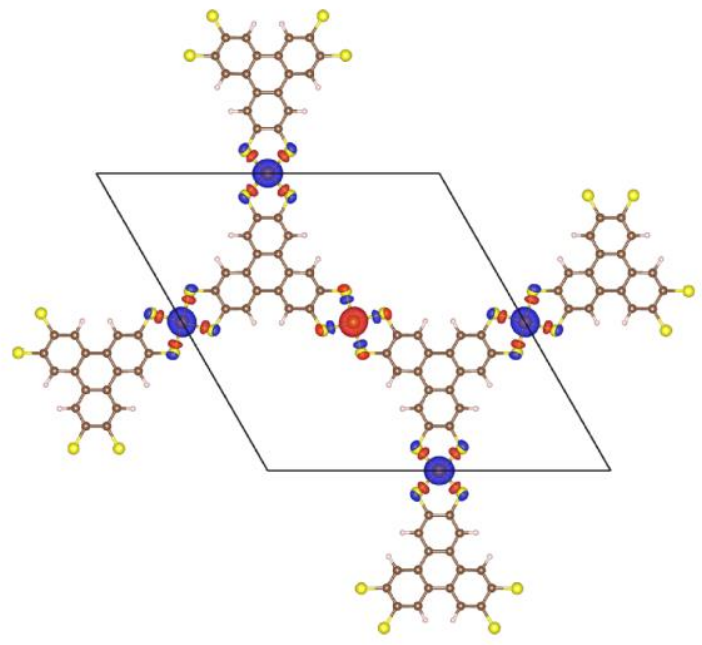

Figure S9. Spin density of the lowest-energy magnetic configuration of the FeTHT framework.

With the more accurate convergence settings used in the electronic-structure calculations, we obtain a magnetic moment of $2.15 \mathrm{BM}$ per formula unit, which is very close to the lowtemperature experimental measurement of 2.2 BM, albeit with a different origin. We also note that the weak intralayer coupling predicted by the calculations would, in principle, allow the system to adopt alternative spin configurations at finite temperature or in response to perturbations to the crystal field, for example oxidation, which is again consistent with experimental observations.

\section{Electronic Structure}

Figure S10 shows the band dispersion and electronic density of states (DoS) curves calculated for the FeTHT framework. As for the CoTHT analogue, the calculations predict this system to be a semi-metal, with a cluster of bands crossing the Fermi energy along the $\Gamma-A$ direction in reciprocal space. This corresponds to the $c$ direction in real space, and an orbitaldensity plot of the states within $25 \mathrm{meV}$ of the Fermi energy (Figure S11) shows that that the partially-occupied bands in both frameworks correspond primarily to chains of interacting $\mathrm{Fe}$ and $\mathrm{S}$ orbitals with a small contribution from the ligand $\pi$ system.

As in our previous study, bands crossing the Fermi energy were identified and the onedimensional $E(k)$ dispersion relation fitted to a quadratic function to estimate the carrier effective masses according to: ${ }^{3}$ 


$$
\frac{1}{m^{*}}=\frac{1}{\hbar^{2}} \frac{\partial^{2} E(k)}{\partial k^{2}}
$$

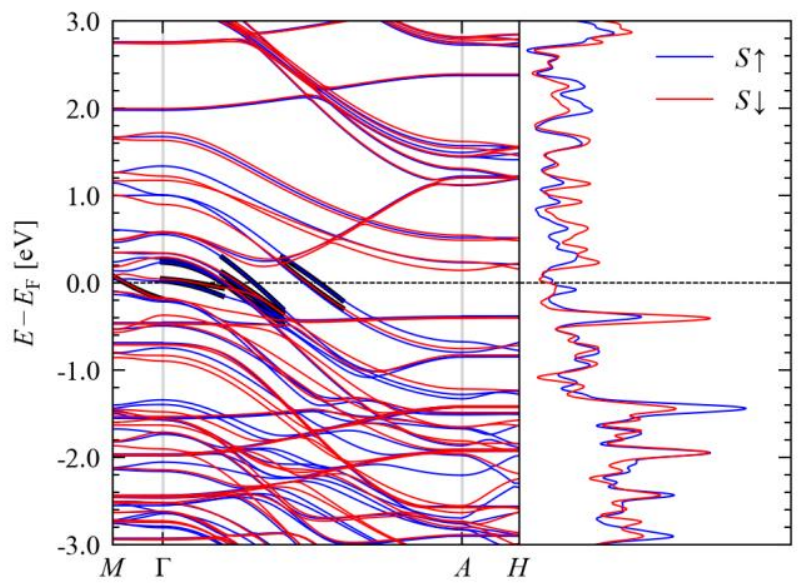

Figure S10. Calculated band dispersion and electronic density of states curves for the FeTHT framework. The blue and red lines denote electronic states in the "up" and "down" spin channels. The thick black lines indicate the parts of the dispersion used to evaluate $\partial^{2} E(k) / \partial k^{2}$ for estimating the carrier effective masses. 


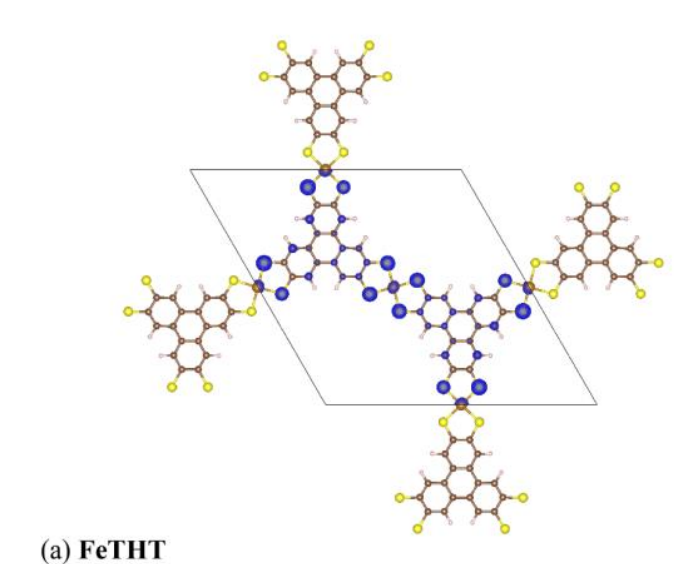

(a) FeTHT

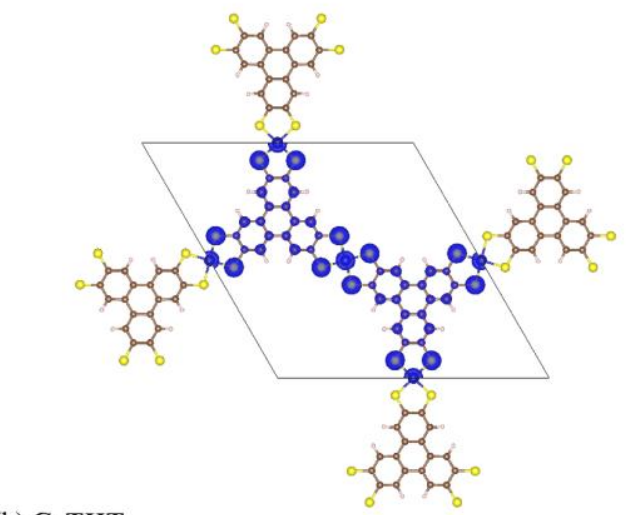

(b) CoTHT

Figure S11. Orbital density showing states within $25 \mathrm{meV}$ of the Fermi energy in the FeTHT (a) and analagous CoTHT (b) framework studied in our previous work (Ref. ${ }^{3}$ ).

The relatively large bandwidth of up to $\sim 1.8 \mathrm{eV}$ results in low carrier effective masses of -0.55 to -6.72 and 1.36 to $2.78 m_{e}$, which can be compared to masses of -0.42 to -1.52 and 0.29 to $8.04 m_{e}$ in CoTHT. ${ }^{3}$ Some of the bands along the $M$ - $\Gamma$ segment, corresponding to in-plane conductivity, are also metallic, with a carrier mass around 0.99 .

\section{Stacking Faults}

Previous studies on the layered $\mathrm{Ni}_{3}(\mathrm{HITP})_{2}$ framework (HITP $=2,3,6,7,10,11$ hexaiminotriphenylenesemiquinonate) showed this system to preferentially adopt a staggered layer arrangement. ${ }^{13}$ In contrast, however, our previous study on CoTHT found that an eclipsed layer configuration was the most energetically favourable. ${ }^{3}$

To examine the layer stacking in the FeTHT framework, we carried out a similar study to explore the potential-energy surface associated with the relative positions of the layers in a bilayer $1 \times 1 \times 2$ supercell expansion (Figure S12). As in our previous work, a series of singlepoint calculations were performed with one layer displaced relative to the other by up to $4 \AA$ 
along the $a$ and $b$ axes, and at each $(\Delta a, \Delta b)$ displacement coordinate the energies of interlayer spacings from 3.084 to $3.584 \AA$ A were calculated and the minimum obtained from a polynomial fit (see Figure S13).

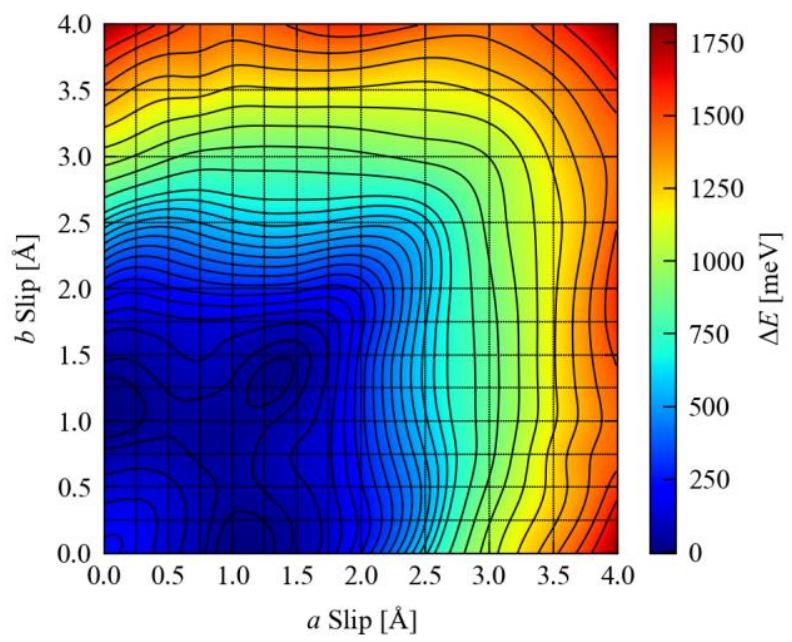

Figure S12. Calculated potential-energy surface associated with layer offsets in the FeTHT framework.

In contrast to the Co analogue, the fully eclipsed configuration of the FeTHT framework is $0.21 \mathrm{eV}$ (34 meV per Fe ion) higher in energy than the global minima, which correspond to displacements along the $a$ or $b$ directions of $\Delta \approx 1.0-1.25 \AA$. There is also a secondary local minimum located close to $\Delta=(1.25,1.25)$ which is a very small $14 \mathrm{meV}(2.4 \mathrm{meV}$ per Fe ion) higher in energy. To investigate further, the two global minima and the low-lying local minimum were fully volume relaxed. Figure S14 shows a view along the $c$ axis of each of the three staggered bilayer structures, and the lattice parameters, magnetic moments and total energies are compared to those of an eclipsed bilayer configuration in Table S2. 


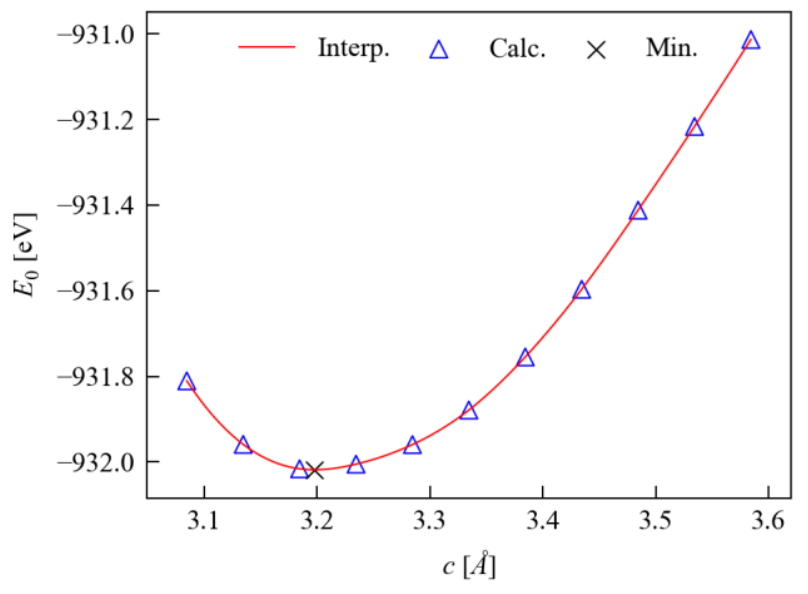

Figure S13. Illustration of the procedure for locating the minimum-energy $c$-axis length (interlayer spacing) at a layer displacement of $\Delta a=1.25$ and $\Delta b=1.25 \AA$. The blue markers show the calculated total energies, the red line shows a $1 \mathrm{D}$ cubic spline interpolation through the data, and the cross marks the position of the identified energy minimum.

\begin{tabular}{lccccc}
\hline & Expt. & Eclipsed & $(1.00,0.00)$ & $(0.00,1.00)$ & $(1.25,0.25)$ \\
\hline$a[\AA]$ & 22.52 & 23.210 & 23.226 & 23.226 & 23.218 \\
\hline$c[\AA]$ & 3.3 & 3.334 & 3.159 & 3.159 & 3.163 \\
$c / a$ & 0.147 & 0.150 & 0.136 & 0.136 & 0.136 \\
\hline$V\left[\AA^{3}\right]$ & 1449 & 1556 & 1476 & 1475 & 1476 \\
$M[\mathrm{BM}]$ & 1.87 & $2.17^{\mathrm{a}}$ & 2.11 & 2.11 & 1.71 \\
\hline $\begin{array}{l}E_{0}[\mathrm{eV} \text { per Fe } \\
\text { ion }]\end{array}$ & - & $-155.30^{\mathrm{a}}$ & -155.39 & -155.39 & -155.40 \\
\hline
\end{tabular}

Table S2. Optimised lattice parameters, total magnetic moments and total energies of the FeTHT framework with eclipsed layers and the three different staggered layer configurations (stacking faults) shown in Figure S14. As in Table 1, The lattice parameters and magnetic moments are given with respect to the crystallographic unit cell, and the total energies are given per Fe ion. The experimental values are shown in the second column for comparison. ${ }^{\text {a }}$ Values computed for a $1 \times 1 \times 2$ expansion of the optimised single-cell model.

After optimisation, the staggered minima are further lowered in energy to $83 \mathrm{meV}$ per Fe ion relative to the eclipsed configuration. The structure displaced along both the $a$ and $b$ axes (Figure S14c) becomes the global minimum, being a comparatively small $15 \mathrm{meV}$ per Fe ion 
lower in energy than the two structures displaced along one of the in-plane directions. All three staggered configurations result in a slight expansion of the $a$ and $b$ axes by 0.01-0.02 $\AA$ and a contraction of the $c$ axis by $0.17-0.18 \AA(5.2 \%)$, which together produce a $5 \%$ reduction in the unit-cell volume. The layer offsets do not appear to change the magnetic ordering, but the calculations predict that simultaneous displacement along both $a$ and $b$ axes could reduce the total magnetic moment by up to $\sim 20 \%$.

(a) $\Delta a=1.00, \Delta b=0.00$

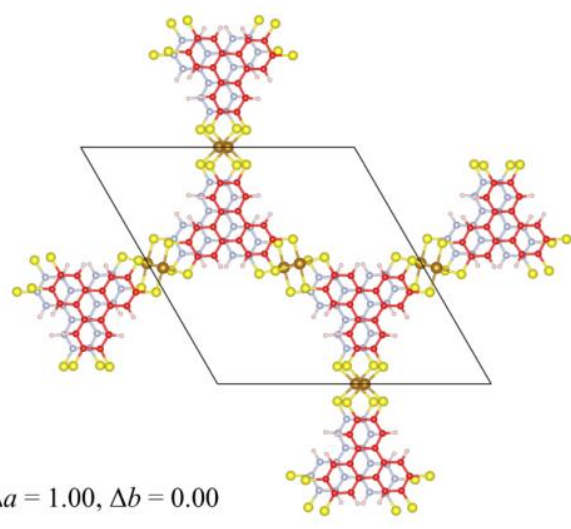

(b) $\Delta a=0.00, \Delta b=1.00$

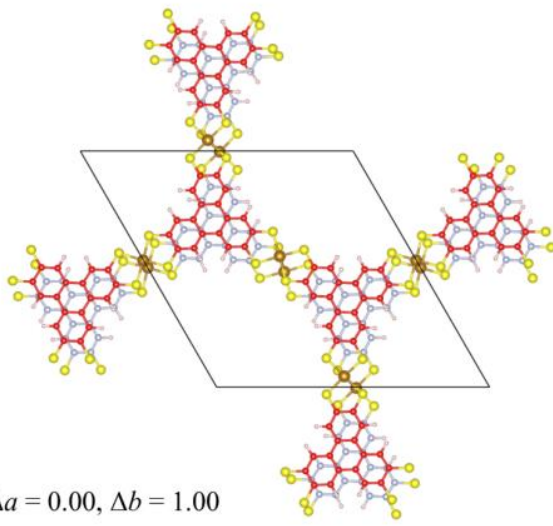

(c) $\Delta a=1.25, \Delta b=1.25$

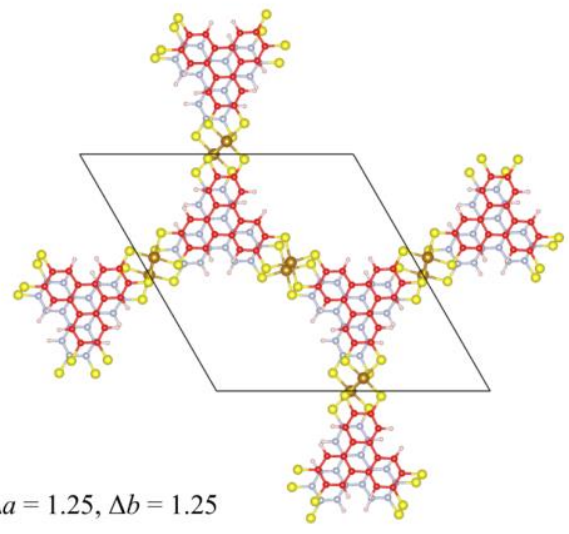

Figure S14. Optimised structures of bilayer models of FeTHT with layer displacements corresponding to the local minima in Fig. 4 , viz. $\Delta=(1.00,0.00)$ (a), $\Delta=(0.00,1.00)$ (b) and $\Delta=$ 
$(1.25,1.25)(\mathrm{c})$. For clarity, the carbon skeletons of the THT ligand in the two layers are coloured red and blue.

The layer displacement has a small effect on the calculated electronic structure (Figure $\mathrm{S} 15$ ), in particular opening a gap in the conduction band at $\sim 1 \mathrm{eV}$ above the Fermi energy, which is similar to the effect of increasing the interlayer spacing in the CoTHT analogue. ${ }^{3}$ To quantify the changes in the density of states around the Fermi energy, we estimated the room-temperature $(T=300 \mathrm{~K})$ concentration of conduction electrons according to:

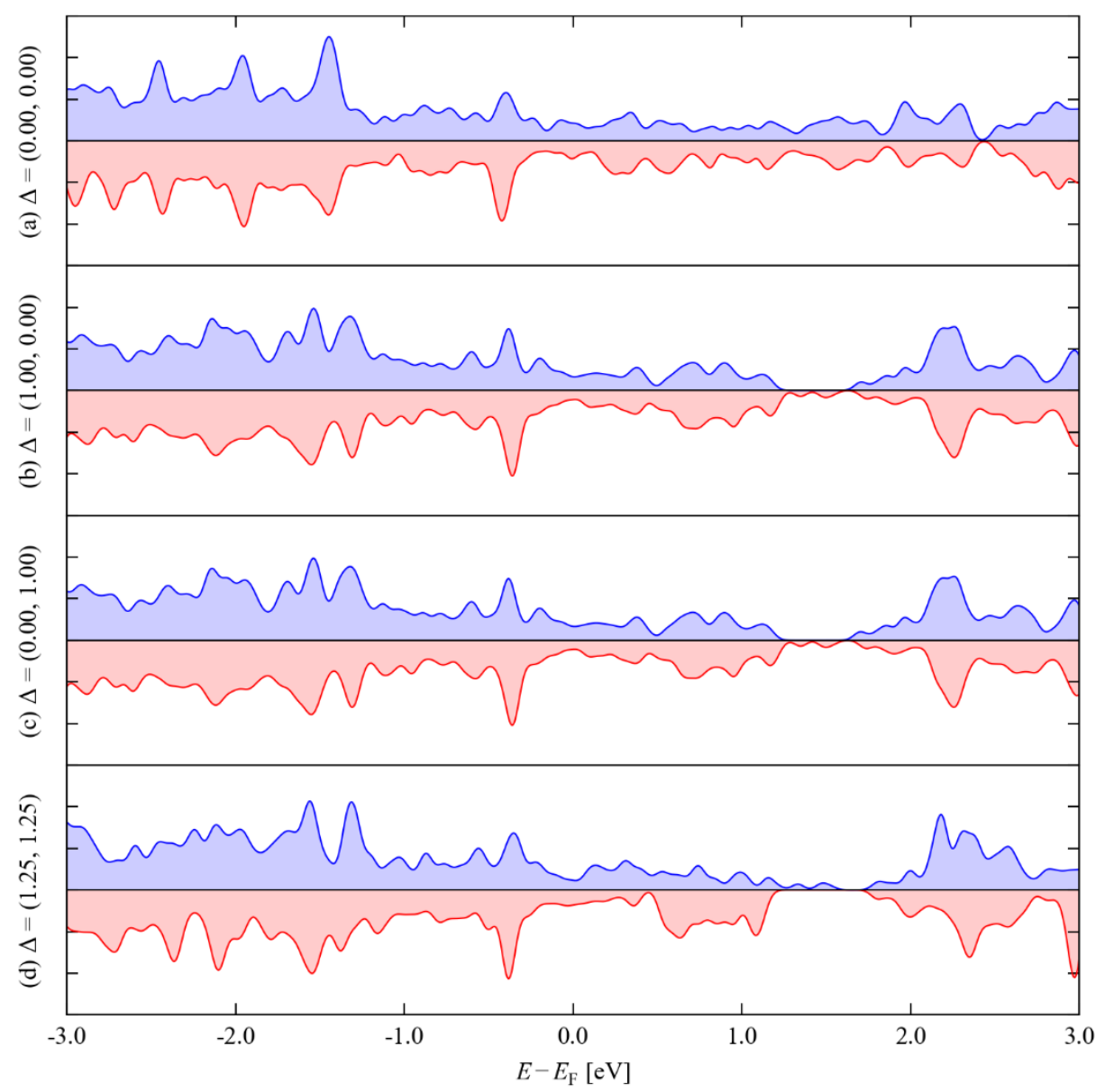

Figure S15. Electronic density of states (DoS) curves of bilayer models of FeTHT in the eclipsed configuration (a) and staggered configurations corresponding to displacements along the $a$ and $b$ axes of $\Delta=(1.00,0.00)(\mathrm{b}), \Delta=(0.00,1.00)$ (c) and $\Delta=(1.25,1.25)$ (d). The positive and negative curves on each subplot denote the DoS in the "up" (blue) and "down" (red) spin channels. 


$$
n_{e}(T)=\int_{E_{\mathrm{F}}}^{E_{\max }} f(E) g(E) d E=\int_{E_{\mathrm{F}}}^{E_{\max }} \frac{1}{1+\exp \left(\frac{E-E_{F}}{k_{\mathrm{B}} T}\right)} g(E) d E
$$

where $f(E)$ is the Fermi-Dirac distribution, $g(E)$ is the electronic density of states, $k_{\mathrm{B}}$ is the Boltzmann constant and the integral runs from the Fermi energy to the highest-energy conduction states in the calculations. We obtained a value of $8.8 \times 10^{25} \mathrm{~m}^{-3}$ for the eclipsed structure, which, compared to a typical value of $10^{28} \mathrm{~m}^{-3}$ for a "good" metal, supports the predicted semi-metallic nature of the FeTHT system. The calculated concentrations of $7.2 \times$ $10^{25}$ and $7.5 \times 10^{25} \mathrm{~m}^{-3}$ for the structures with layers displaced along the $a / b$ and both axes, respectively, suggest that layer misalignment reduces the density of conduction states close to the Fermi energy, as would be expected given the nature of the conductive states (c.f. Figure S6). This result is again similar to the behaviour of the CoTHT analogue. ${ }^{3}$

These results indicate that the FeTHT system may show a propensity for layer misalignment, in contrast to the CoTHT analogue, and the effect of this, together with environmental factors such as the presence of guest molecules in the pores, may play a role in the measured temperature dependence of the resistivity.

\section{Effect of a Hubbard U Correction}

Systems with strongly-localised electrons, such as those containing $d$ - and f-block elements, can pose a challenge to (semi-)local DFT methods such as PBEsol, where selfinteraction error tends to overly delocalise the electrons and predict unrealistic electron energies and other calculated physical properties such as the magnetic ground state. ${ }^{14}$ Of particular importance to this work is that in some systems the error can lead to calculations predicting insulators to be metals. ${ }^{15}$ A straightforward and computationally-efficient method to correct for self-interaction is to apply a Hubbard $U$ correction to specific atomic states and introduce an energy penalty to force the orbital occupations towards integer values. ${ }^{8,14}$

To test the effect of a Hubbard correction on our results, we performed an identical set of calculations on the single-cell model of the FeTHT framework with a Hubbard correction of $U=$ $5 \mathrm{eV}$ applied to the $\mathrm{Fe} \mathrm{d}$ states. This resulted in a slight lengthening of the $a$ and $c$ lattice constants by $\sim 0.1 \AA$ and a $1 \%$ increase in the cell volume (Table S3). The frustrated antiferromagnetic configuration holds, but stronger localisation of the Fe d electrons increases the total magnetic moment from 2.2 to $2.4 \mathrm{BM}$ per F.U., with the individual Fe moments increasing from \pm 2.0 to $\pm 2.8 \mathrm{BM}$ per ion. The correction leads to noticeable changes in the electronic structure (Figure S16), but the semi-metallic nature of the framework is preserved, and the carrier effective masses obtained from the curvature of bands along the $M-\Gamma$ and $\Gamma-A$ directions are of comparable magnitude to the bare DFT values, albeit with larger variation and heavier extremes. 
Similar DFT $+U$ calculations on the CoTHT analogue with an identical correction of $U=$ $5 \mathrm{eV}$ applied to the Co d states gave similar results (Table 4, Figure S17), leading to expansion of the $c$ axis by $\sim 0.3 \AA$, a $9.5 \%$ increase in the cell volume, and an increase in the total and Co magnetic moments from 2.2 to $4.8 \mathrm{BM}$ per F.U. and 2.0 to $2.8 \mathrm{BM}$ per Co ion, respectively. The larger changes compared to the FeTHT framework could be due to the predicted fullyferromagnetic ground state in the Co framework, although systematic studies on transition-metal oxides suggest that the Co system may not need as large a $U$ value as the $\mathrm{Fe}$ one. ${ }^{16}$ Once again, despite noticeable changes to the electronic band dispersion and DoS, the predicted semimetallic electronic structure is preserved, and the calculated carrier effective masses are of a similar magnitude.

These calculations provide some confidence that the predicted semi-metallic electronic structure of the pristine framework is not an artefact from our use of the semi-local PBEsol exchange-correlation functional, although we note that more sophisticated treatments of electron correlation may produce a different picture. However, we consider such an undertaking to be beyond the scope of this investigation.

\begin{tabular}{lccc}
\hline & & \multicolumn{2}{c}{ Calc. } \\
\cline { 3 - 4 } & Expt. & PBEsol-D3 & PBEsol-D3 $+U$ \\
\hline$a[\AA]$ & 22.52 & 23.210 & 23.308 \\
\hline$c[\AA]$ & 3.3 & 3.334 & 3.342 \\
$c / a$ & 0.147 & 0.150 & 0.143 \\
$V\left[\AA^{3}\right]$ & 1449 & 1556 & 1572 \\
\hline & & 2.15 & 2.43 \\
$M[\mathrm{BM}]$ & 2.20 & $(1.95,1.95,-$ & $(2.83,2.83,-$ \\
& & $1.95)$ & $2.83)$ \\
\hline
\end{tabular}

Table S3. Calculated lattice parameters and magnetic moments of the FeTHT framework structure obtained using the PBEsol-D3 functional with and without a Hubbard correction of $U=$ $5 \mathrm{eV}$ applied to the Fe d states. For each calculation, both the total magnetic moment and the moments of the three Fe ions are given. Experimental values are shown in the second column for comparison. 

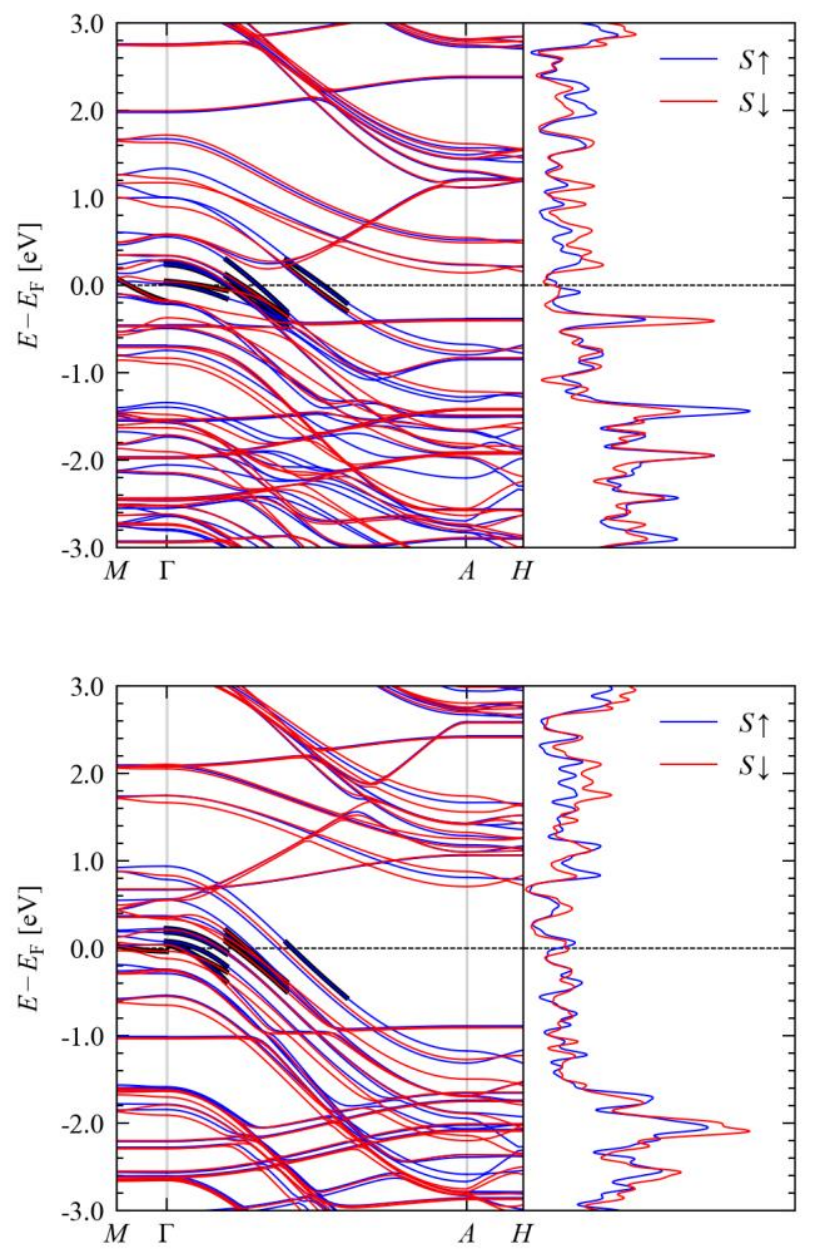

Figure S16. Band dispersion and electronic density of states (DoS) curves of the FeTHT framework calculated with PBEsol (top) and PBEsol $+U$ with a Hubbard correction of $U=5 \mathrm{eV}$ applied to the Fe d states (bottom). As in Figure S5, the blue and red colours denote electronic states in the two spin channels, and the thick black lines indicate parts of the dispersion used to evaluate $\partial^{2} E(k) / \partial k^{2}$ for estimating the carrier effective masses. 


\begin{tabular}{lccc}
\hline & & \multicolumn{2}{c}{ Calc. } \\
\cline { 3 - 4 } & Expt. & PBEsol-D3 & PBEsol-D3 $+U$ \\
\hline$a[\AA]$ & 22.52 & 23.133 & 23.135 \\
$c[\AA]$ & 3.3 & 3.140 & 3.438 \\
\hline$c / a$ & 0.147 & 0.136 & 0.149 \\
\hline$V\left[\AA^{3}\right]$ & 1449 & 1455 & 1593 \\
\hline$M[\mathrm{BM}]$ & & & 4.77 \\
& 1.87 & 2.15 & $(1.81,1.81,1.81)$ \\
\hline
\end{tabular}

Table S4. Calculated lattice parameters and magnetic moments of the CoTHT analogue of the FeTHT framework obtained using the PBEsol-D3 functional with and without a Hubbard correction of $U=5 \mathrm{eV}$ applied to the Co d states. For each calculation, both the total magnetic moment and the moments of the three Co ions are given. Experimental values are shown in the second column for comparison. 

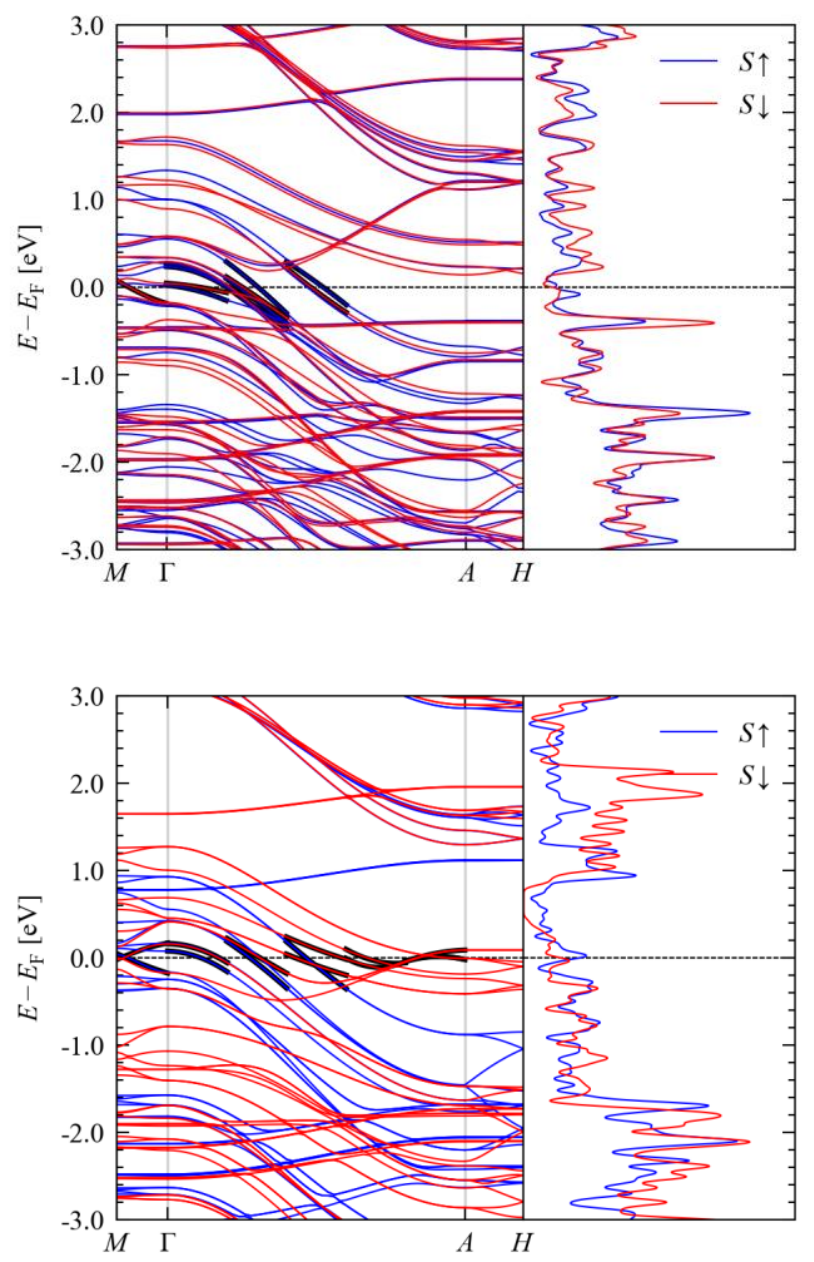

Figure S17. Band dispersion and electronic density of states (DoS) curves of the Co analogue of the FeTHT framework calculated with PBEsol (top) and PBEsol+ $U$ with a Hubbard correction of $U=5 \mathrm{eV}$ applied to the Fe d states (bottom). As in Figure S5, the blue and red colours denote electronic states in the two spin channels, and the thick black lines indicate parts of the dispersion used to evaluate $\partial^{2} E(k) / \partial k^{2}$ for estimating the carrier effective masses. 
Table S5. Peak parameters to fit $\mathrm{Fe}^{2+}$ and $\mathrm{Fe}^{3+}$ multiplets in FeTHT. The reference parameters are of $\mathrm{Fe}_{3} \mathrm{O}_{4} .{ }^{4} \mathrm{Fe}_{3} \mathrm{O}_{4}$ has a mixed $\mathrm{Fe}^{3+/ 2+}$ valency with a 2:1 ratio, which is comparable to the FeTHT and thus, the primary reason for reference in fitting the $\mathrm{Fe} 2 \mathrm{p}$ region. Details of the fitting can be found in the table below. Peak 1 represents the lowest binding energy peak for both the $\mathrm{Fe}^{2+}$ and $\mathrm{Fe}^{3+}$ multiplets. Note that the single $\mathrm{Fe}^{3+}$ satellite $(\sim 716 \mathrm{eV})$ is not in the table because peak constraints were not held fixed between samples.

\begin{tabular}{|c|c|c|c|c|c|c|c|c|c|c|c|}
\hline & $\begin{array}{c}\text { Peak 1 (eV) } \\
\text { [FWHM] }\end{array}$ & $\%$ & $\begin{array}{c}\text { Peak } 2(\mathrm{eV}) \\
{[\mathrm{FWHM}]}\end{array}$ & $\%$ & $\begin{array}{c}\Delta \mathrm{E}_{(\text {peak2-peak1) }} \\
(\mathrm{eV})\end{array}$ & $\begin{array}{c}\text { Peak } 3(\mathrm{eV}) \\
{[\text { FWHM] }}\end{array}$ & $\%$ & $\begin{array}{c}\Delta \mathrm{E}_{(\text {peak3-peak2) }} \\
(\mathrm{eV})\end{array}$ & $\begin{array}{c}\text { Peak } 4(\mathrm{eV}) \\
\text { [FWHM] }\end{array}$ & $\%$ & $\begin{array}{c}\Delta \mathrm{E}_{(\text {peak4-peak3) }} \\
(\mathrm{eV})\end{array}$ \\
\hline \multicolumn{12}{|l|}{$\mathrm{Fe} 2+$} \\
\hline Reference & $708.3[1.2]$ & 41.6 & $709.3[1.2]$ & 43.2 & 1.0 & $710.4[1.4]$ & 15.2 & 1.1 & & & \\
\hline Pristine & $708.2[1.0]$ & 41.8 & $709.2[1.6]$ & 43.1 & 1 & $710.3[1.6]$ & 15.1 & 1.1 & & & \\
\hline \multicolumn{12}{|l|}{$\mathrm{Fe} \mathrm{3+}$} \\
\hline Reference & $710.2[1.4]$ & 34.9 & $711.1[31.8]$ & 31.8 & 1.1 & $712.4[1.4]$ & 22.6 & 1.1 & $713.6[1.4]$ & 10.8 & 1.2 \\
\hline Pristine & 710.1 [1.6] & 35.1 & $711.2[1.5]$ & 31.9 & 1.1 & $712.3[1.6]$ & 22.5 & 1.1 & $713.5[1.6]$ & 10.5 & 1.2 \\
\hline 7 Days & $710.1[1.6]$ & 35.1 & $711.2[1.5]$ & 31.9 & 1.1 & $712.3[1.6]$ & 22.5 & 1.1 & $713.5[1.6]$ & 10.5 & 1.2 \\
\hline
\end{tabular}
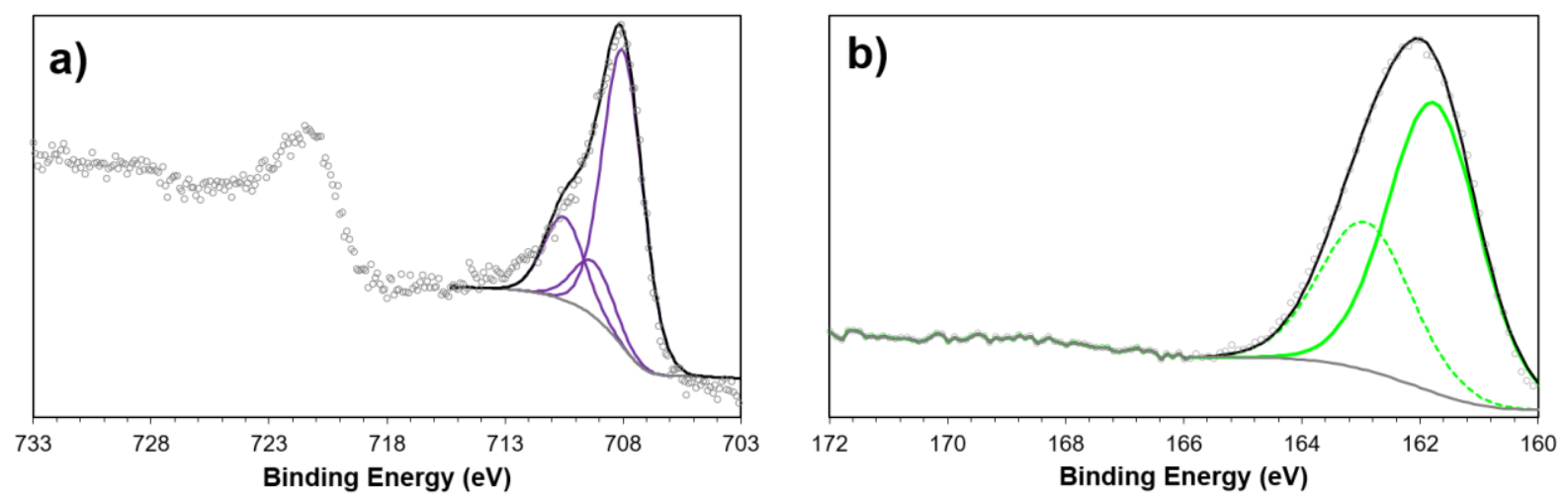

Figure S18. XPS spectra showing the iron $2 p$ (a) and sulfur $2 p$ (b) features from the $\left[\mathrm{Fe}(\mathrm{bdt})_{2}\right]\left[\mathrm{HNEt}_{3}\right]_{2}$ molecular complex, where bdt $=1,2$-benzenedithiolate.
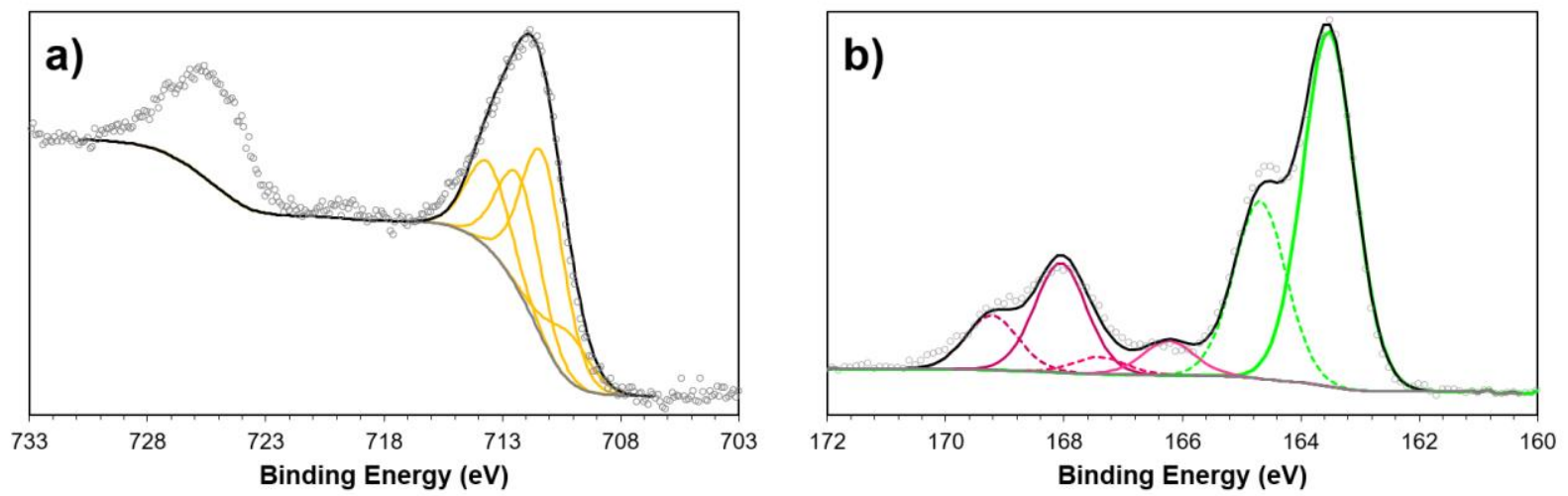

Figure S19. XPS spectra showing the iron $2 p$ (a) and sulfur $2 p$ (b) features from the $\left[\mathrm{Fe}(\mathrm{bdt})_{2}\right]\left[\mathrm{HNEt}_{3}\right]_{2}$ molecular complex after 3 days of oxidation in air, where bdt $=1,2-$ benzenedithiolate. 

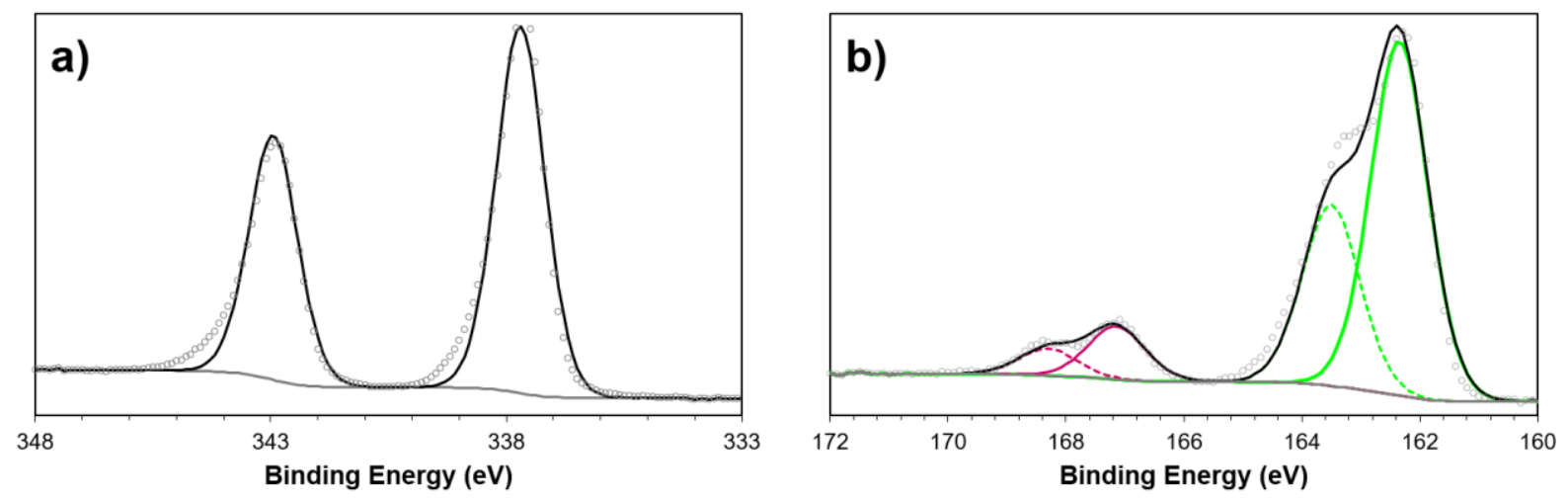

Figure S20. XPS spectra showing the palladium $3 d$ (a) and sulfur $2 p$ (b) features of a $\left[\mathrm{Pd}(\mathrm{bdt})_{2}\right] \mathrm{K}_{2}$ molecular complex after 30 minutes of air exposure, where bdt $=1,2-$ benzenedithiolate.
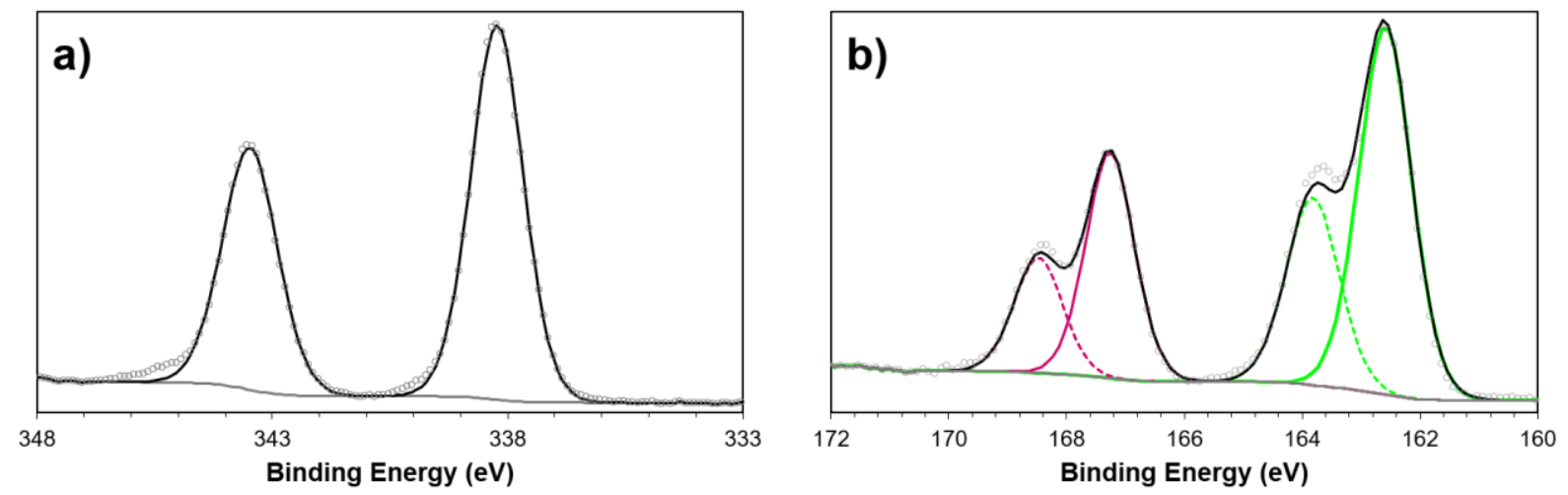

Figure S21. XPS spectra showing the palladium $3 d$ (a) and sulfur $2 p$ (b) features of a $\left[\mathrm{Pd}(\mathrm{bdt})_{2}\right] \mathrm{K}_{2}$ molecular complex after 3 days of air exposure, where bdt = 1,2-benzenedithiolate. The molecular formula of this crystalline coordination polymer is $\left\{\left[\mathrm{K}_{4}(\text { thf })_{4}\left(\mathrm{H}_{2} \mathrm{O}\right)_{2.28}\right][\mathrm{Pd}-\right.$ $\left.\left.\left(\mathrm{O}_{2} \mathrm{SC}_{6} \mathrm{H}_{4} \mathrm{~S}\right)_{1.36}\left(\mathrm{OSC}_{6} \mathrm{H}_{4} \mathrm{~S}\right)_{0.64}\right]_{2}\right\}_{\mathrm{n}}$, as reported by Zamora and co-workers. ${ }^{17}$ 
a)

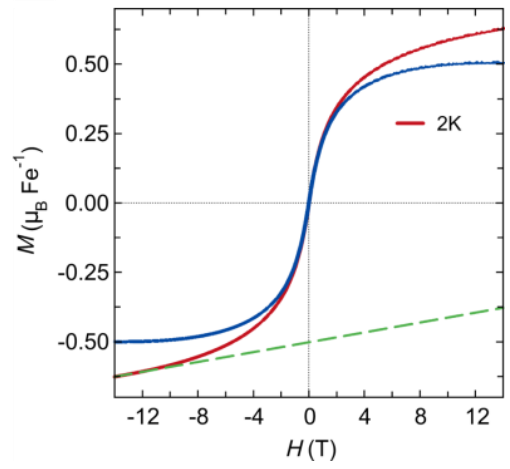

b)

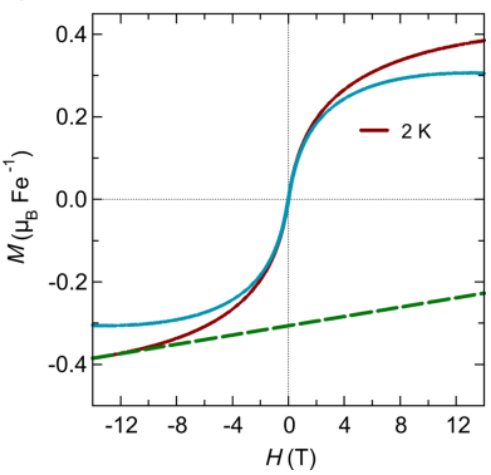

Figure S22. Magnetic hysteresis loops in an FeTHT sample exposed to air for three days (a) and pristine FeTHT (b).

a)

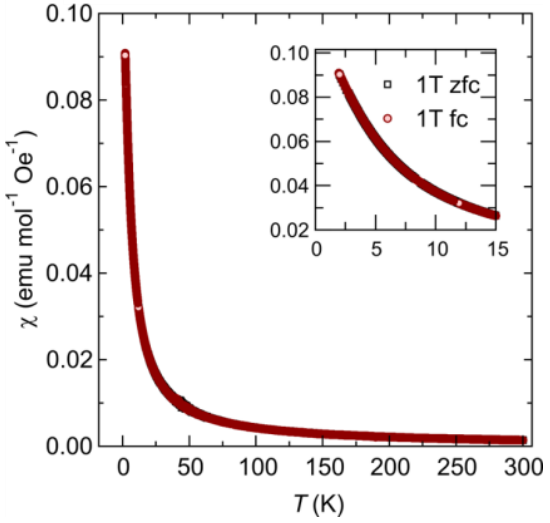

b)

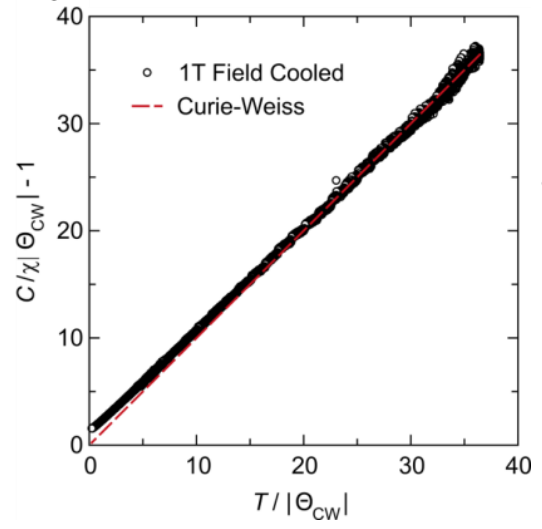

c)

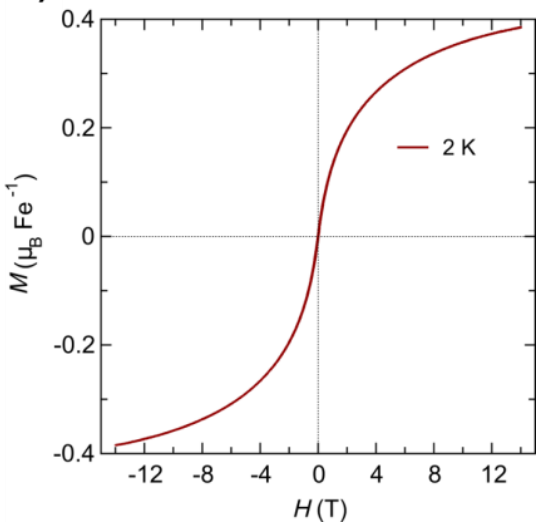

Figure S23. Magnetic studies of pristine FeTHT. (a) temperature-dependent susceptibility, $(b)$ Curie-Weiss fit of the high-temperature region (200-300 K), and (c) hysteresis loop collected at $2 \mathrm{~K}$.

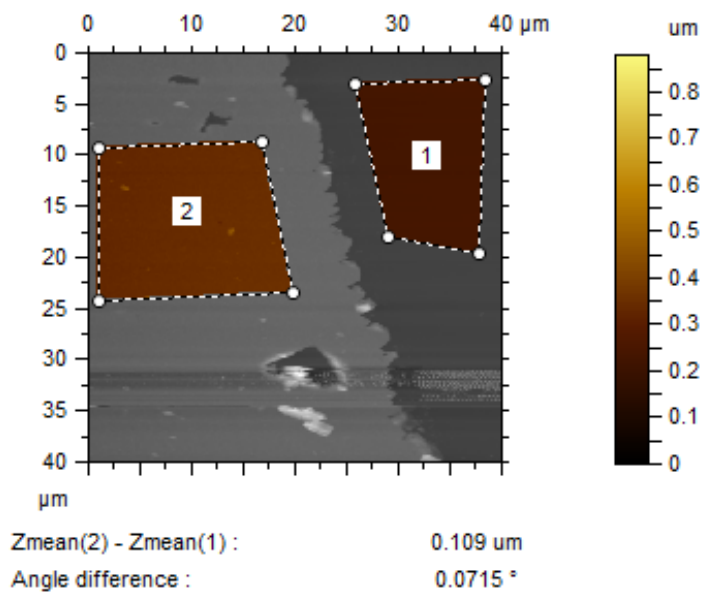

Figure S24. Typical Atomic Force Microscopy (AFM) studies of FeTHT films. The measured thickness of the FeTHT film is 109(11) nm. 


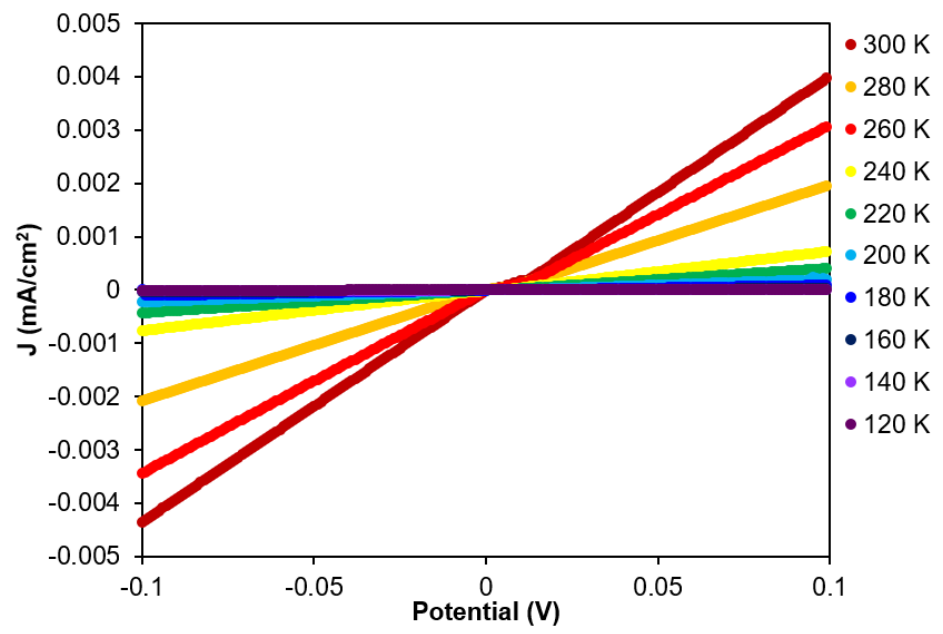

Figure S25. Variable-temperature I-V traces of FeTHT demonstrating Ohmic behavior.

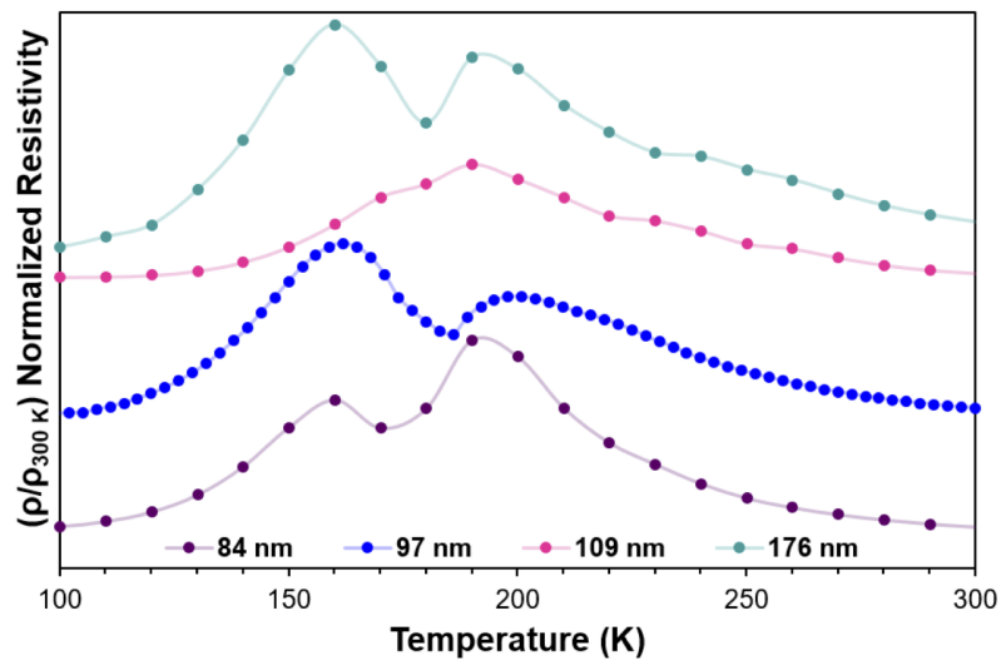

Figure S26. Overlay of the temperature-dependent resistivity data for FeTHT films with thicknesses ranging from $84(8)$ to $176(18) \mathrm{nm}$. 

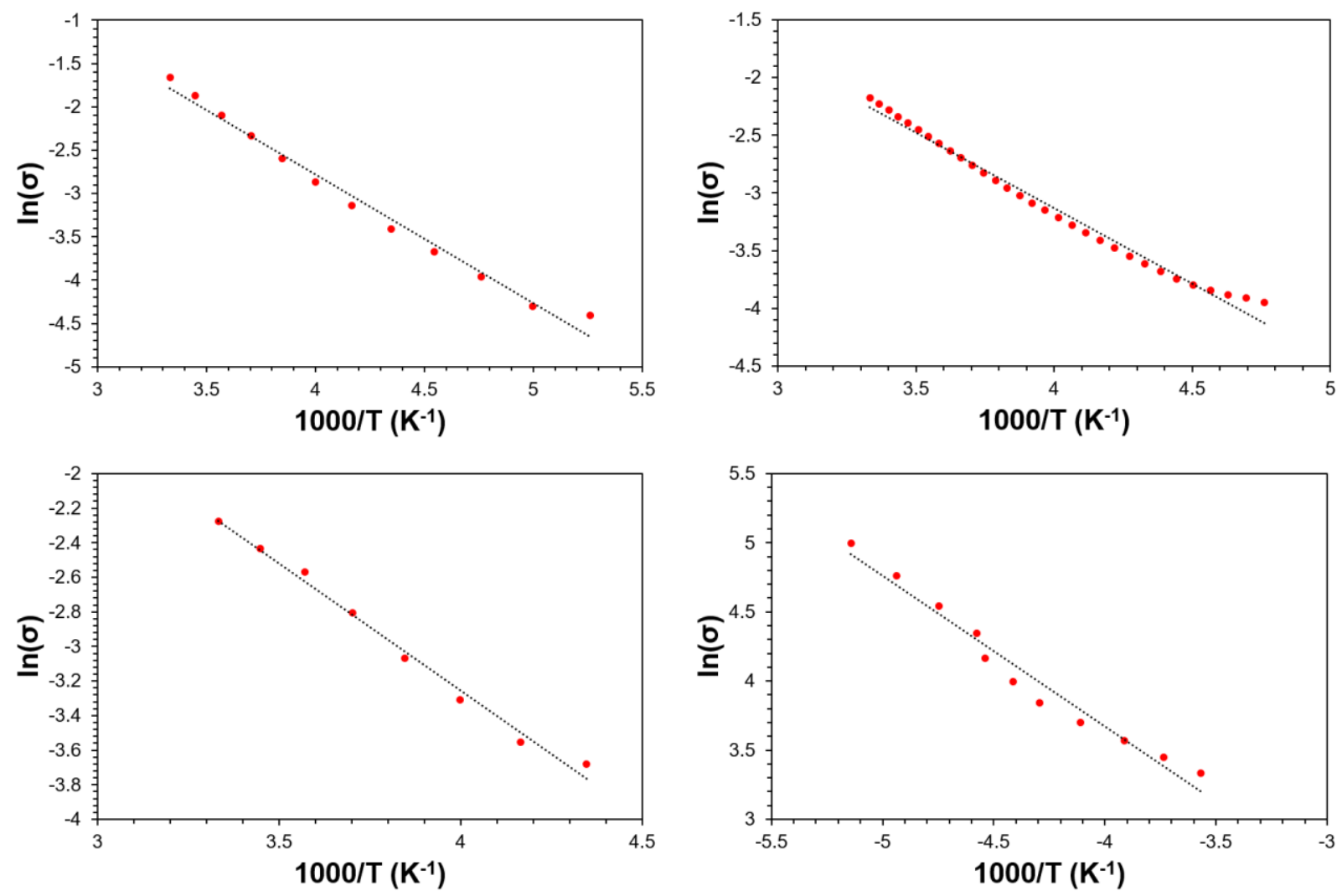

Figure S27. Arrhenius plots for FeTHT films with thicknesses of 84(8) nm (top left), 97(10) nm (top right), 109(11) nm (bottom left), and 176(18) nm (bottom right). The data was fit from $300-$ $230 \mathrm{~K}$.

Recent DFT studies and a related examination of the temperature-dependent resistivity data for the $\mathrm{Ni}_{3}(\mathrm{HITP})_{2}$ framework have suggested that the Mott variable-range hopping model best describes the conductivity behaviour of this material. ${ }^{18}$ Fitting the high-temperature data for FeTHT to the Mott variable-range hopping model and generating an Arrhenius plot gives an activation energy for charge hopping ranging between 6.2 and $8.9 \mathrm{meV}$ (Figure S28; Table S6). Interestingly, these values are similar to the $6 \mathrm{meV}$ hopping barrier reported for the $\mathrm{Ni}_{3}(\mathrm{HITP})_{2} 2 \mathrm{D}$ framework. This small activation barrier suggests that defects, likely grain boundaries in the $2 \mathrm{D}$ sheets, play a significant role in the transport properties of these 2D MOFs. 

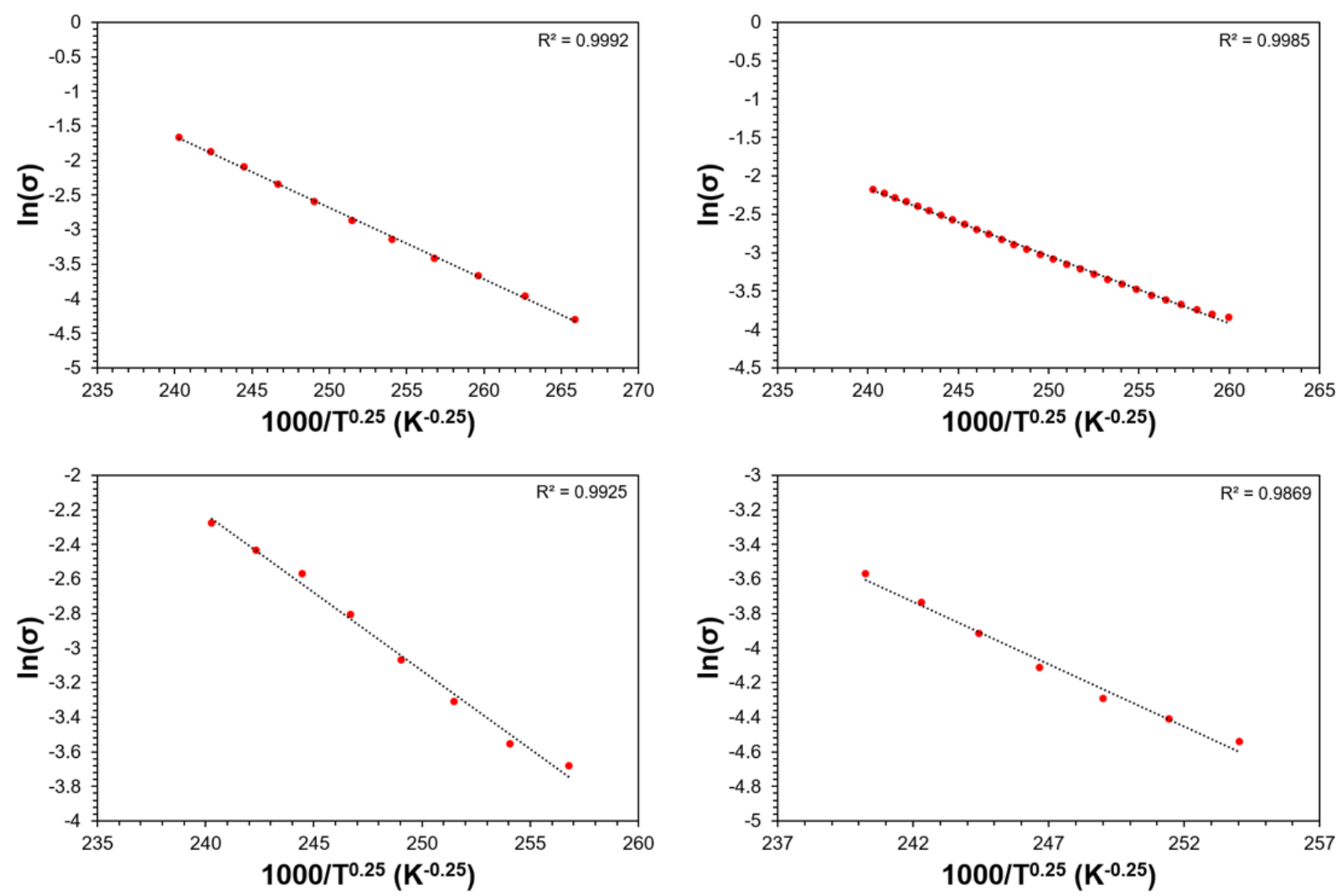

Figure S28. Arrhenius plots using the variable range hopping model for FeTHT films with thicknesses of 84(8) nm (top left), 97(10) nm (top right), 109(11) nm (bottom left), and 176(18) $\mathrm{nm}$ (bottom right). The data was fit from $300-230 \mathrm{~K}$.
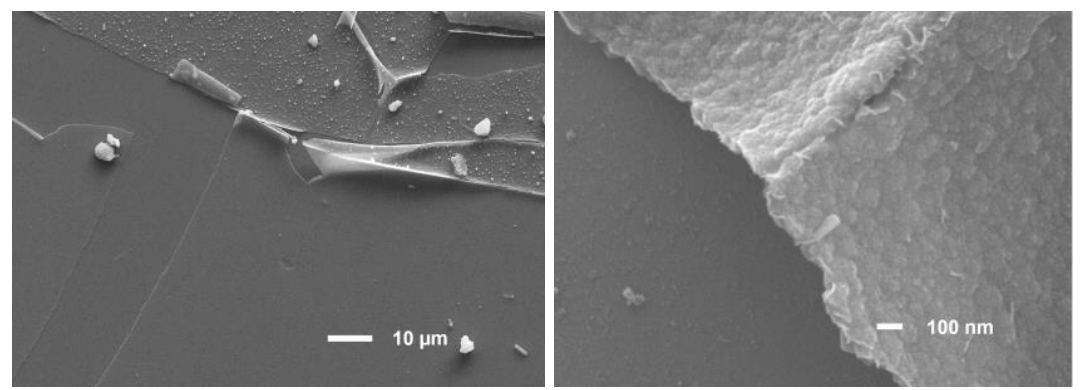

Figure S29. SEM images of FeTHT after resistivity studies. (left) medium magnification $(1000 \times)$ and (right) high magnification $(60,000 \times)$. 
Table S6: Summary of electrical transport data for FeTHT films.

\begin{tabular}{cccccc}
\hline Entry & Thickness $(\mathrm{nm})$ & Conductivity $(\mathrm{S} / \mathrm{cm}) @ 300 \mathrm{~K}$ & $\mathrm{E}_{\mathrm{a}}^{[a]}(\mathrm{meV})$ & $\mathrm{E}_{\mathrm{a}}^{[\mathrm{b}]}(\mathrm{meV})$ & $\mathrm{T}_{\text {Metallic }}(\mathrm{K})$ \\
\hline $\mathbf{1}$ & $84(8)$ & 0.3 & 12.8 & 8.9 & 160 \\
$\mathbf{2}$ & $97(10)$ & 0.1 & 11.3 & 7.5 & 190 \\
$\mathbf{3}$ & $109(11)$ & 0.1 & 12.7 & 7.8 & 160 \\
$\mathbf{4}$ & $176(18)$ & 0.03 & 9.4 & 6.2 & 160 \\
$\mathbf{5}$ & $410(41)$ & 0.02 & - & 8.5 & 170 \\
\hline
\end{tabular}

${ }^{[a]}$ fitted using the Arrhenius equation; ${ }^{[b]}$ fitted using the variable range hopping model.

Table S7: Transport data for an FeTHT sample with 275(28) nm thickness after exposure to ambient atmosphere for varying amount of time (from Figure 5).

\begin{tabular}{|ccc} 
Air Exposure & Conductivity $(\mathbf{S} / \mathbf{c m}) @ \mathbf{3 0 0}$ & $\mathbf{T}_{\text {metallic }}(\mathbf{K})$ \\
\hline as-prepared & 0.2 & 100 \\
2.5 days & 0.02 & 135 \\
1 month & $1.4 \times 10^{-3}$ & 265 \\
1.5 months & $8.0 \times 10^{-4}$ & 300
\end{tabular}

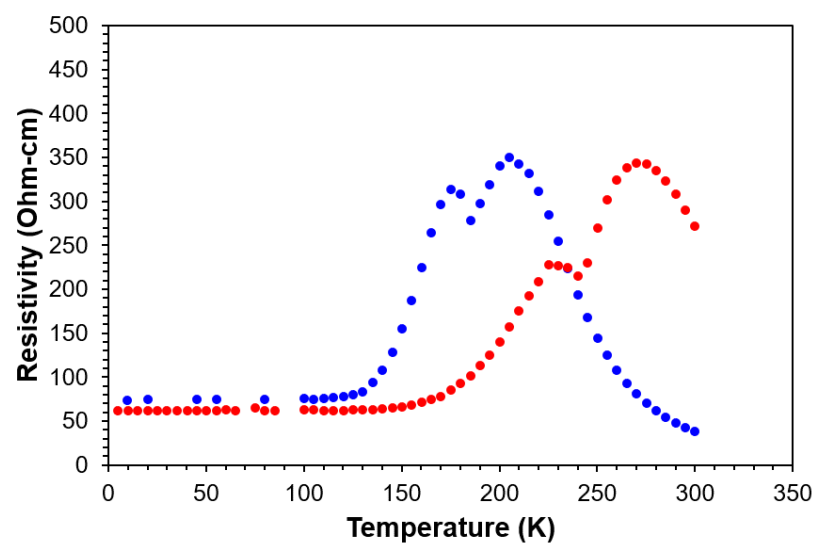

Figure S30. Temperature-dependent resistivity data for a 310(31) nm FeTHT film as-prepared (blue) and after 3 days of air exposure at $60^{\circ} \mathrm{C}$ (red). 


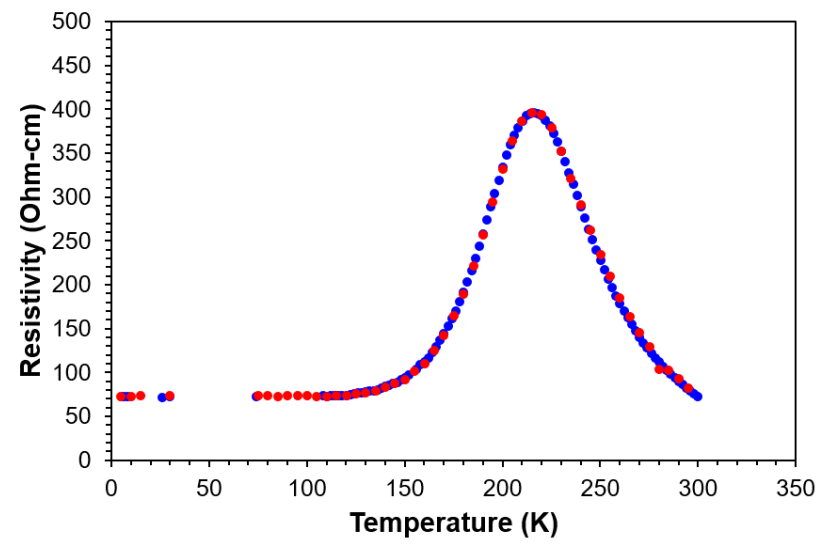

Figure S31. Temperature-dependent resistivity data for a 310(31) nm FeTHT film as-prepared (blue) and after 3 days at $60^{\circ} \mathrm{C}$ under 1 atmosphere of helium (red).

Table S8. Comparison of the structural and physical properties of two FeTHT 2D MOFs

\begin{tabular}{|c|c|c|}
\hline & $\mathrm{Fe}_{3}(\mathrm{THT})_{2}\left(\mathrm{NH}_{4}\right)_{3}($ reference 19$)$ & FeTHT as-prepared (this work) \\
\hline Unit cell & $\begin{aligned} \mathrm{a}=\mathrm{b} & =\sim 1.97 \mathrm{~nm} \\
\mathrm{c} & =\sim 3.3 \AA\end{aligned}$ & $\begin{array}{c}\mathrm{a}=\mathrm{b}=\sim 1.9 \mathrm{~nm} \\
\mathrm{c}=\sim 3.34 \AA\end{array}$ \\
\hline Stacking mode & Inclined AA & Inclined AA \\
\hline $\mathrm{Fe}^{2+}$ & $<0.1 \%$ & $67 \%$ \\
\hline $\mathrm{Fe}^{3+}$ & $\sim 100 \%$ & $33 \%$ \\
\hline BET surface area & $526 \mathrm{~m}^{2} / \mathrm{g}$ & $370 \mathrm{~m}^{2} / \mathrm{g}$ \\
\hline Transport type & semiconducting & semiconducting / metallic \\
\hline
\end{tabular}

\section{References:}

(1) Sakamoto, R.; Kambe, T.; Tsukada, S.; Takada, K.; Hoshiko, K.; Kitagawa, Y.; Okumura, M.; Nishihara, H. $\pi$-Conjugated Trinuclear Group-9 Metalladithiolenes with a Triphenylene Backbone. Inorg. Chem. 2013, 52, 7411-7416.

(2) Clough, A. J.; Yoo, J. W.; Mecklenburg, M. H.; Marinescu, S. C. Two-Dimensional Metal-Organic Surfaces for Efficient Hydrogen Evolution from Water. J. Am. Chem. Soc. 2015, 137, 118-121.

(3) Clough, A. J.; Skelton, J. M.; Downes, C. A.; de la Rosa, A. A.; Yoo, J. W.; Walsh, A.; Melot, B. C.; Marinescu, S. C. Metallic Conductivity in a Two-Dimensional Cobalt Dithiolene MetalOrganic Framework. J. Am. Chem. Soc. 2017, 139, 10863-10867.

(4) Grosvenor, A. P.; Kobe, B. A.; Biesinger, M. C.; McIntyre, N. S. Investigation of multiplet splitting of Fe 2p XPS spectra and bonding in iron compounds. Surf. Interface Anal. 2004, 36, 1564-1574.

(5) Kresse, G.; Hafner, J. Ab initio molecular dynamics for liquid metals. Phys. Rev. B 1993, 47, 558(R)-561(R). 
(6) Perdew, J. P.; Ruzsinszky, A.; Csonka, G. I.; Vydrov, O. A.; Scuseria, G. E.; Constantin, L. A.; Zhou, X.; Burke, K. Restoring the Density-Gradient Expansion for Exchange in Solids and Surfaces. Phys. Rev. Lett. 2008, 100, 136406.

(7) Grimme, S.; Antony, J.; Ehrlich, S.; Krieg, H. A consistent and accurate ab initio parametrization of density functional dispersion correction (DFT-D) for the 94 elements H-Pu. J. Chem. Phys. 2010, 132, 154104.

(8) Dudarev, S. L.; Botton, G. A.; Savrasov, S. Y.; Humphreys, C. J.; Sutton, A. P. Electronenergy-loss spectra and the structural stability of nickel oxide: An LSDA+U study. Phys. Rev. B 1998, $57,1505-1509$.

(9) Blöchl, P. E. Projector augmented-wave method. Phys. Rev. B 1994, 50, 17953-17979.

(10) Kresse, G.; Joubert, D. From ultrasoft pseudopotentials to the projector augmented-wave method. Phys. Rev. B 1999, 59, 1758-1775.

1976, 13 .

(11) Monkhorst, H. J.; Pack, J. D. Special points for Brillouin-zone integrations. Phys. Rev. B

(12) Skelton, J. M. Transformer. 2017.

(13) Sheberla, D.; Sun, L.; Blood-Forsythe, M. A.; Er, S.; Wade, C. R.; Brozek, C. K.; Aspuru-Guzik, A.; Dincă, M. High Electrical Conductivity in $\mathrm{Ni}_{3}(2,3,6,7,10,11 \text {-hexaiminotriphenylene })_{2}$, a Semiconducting Metal-Organic Graphene Analogue. J. Am. Chem. Soc. 2014, 136, 8859-8862.

(14) Shick, A. B.; Liechtenstein, A. I.; Pickett, W. E. Implementation of the LDA+U method using the full-potential linearized augmented plane-wave basis. Phys. Rev. B 1999, 60, 10763-10769.

(15) Sheykhi, S.; Payami, M. Electronic structure properties of $\mathrm{UO}_{2}$ as a Mott insulator. Physica C 2018, 549, 93-94.

(16) Wang, L.; Maxisch, T.; Ceder, G. Oxidation energies of transition metal oxides within the GGA + U framework. Phys. Rev. B 2006, 73, 195107.

(17) Castillo, O.; Delgado, E.; Goméez-García, C. J.; Hernańdez, D.; Hernańdez, E.; Herrasti, P.; Martín, A.; Zamora, F. i. Comparative Studies of Oxidation Processes on Group 10 Metals Dithiolene Derivatives in the Formation of Coordination Polymers. Cryst. Growth Des. 2018, 18, 2486-2494.

(18) Foster, M. E.; Sohlberg, K.; Allendorf, M. D.; Talin, A. A. Unraveling the Semiconducting/Metallic Discrepancy in $\mathrm{Ni}_{3}(\mathrm{HITP})_{2}$. J. Phys. Chem. Lett. 2018, 9, 481-486.

(19) Dong, R.; Han, P.; Arora, H.; Ballabio, M.; Karakus, M.; Zhang, Z.; Shekhar, C.; Adler, P.; Petkov, P. S.; Erbe, A.; Mannsfeld, S. C. B.; Felser, C.; Heine, T.; Bonn, M.; Feng, X.; Cánovas, E. High-mobility band-like charge transport in a semiconducting two-dimensional metal-organic framework. Nat. Mater. 2018, 17, 1027-1032. 